\title{
Asymptotic Stability and Boundedness of Stochastic Functional Differential Equations with Markovian Switching
}

\author{
Lichao Feng ${ }^{a, b}$, Shoumei $\mathrm{Li}^{a}{ }^{*}$ and Xuerong $\mathrm{Mao}^{c}$ \\ ${ }^{a}$ College of Applied Sciences, Beijing University of Technology, Beijing, 100124, P.R. China \\ ${ }^{b}$ College of Science, North China University of Science and Technology, Hebei, 063009, P.R. China \\ ${ }^{c}$ Department of Mathematics and Statistics, University of Strathclyde, Glasgow, G1 1XH, UK \\ e-mail address: fenglichao19820520@163.com, lisma@bjut.edu.cn, x.mao@strath.ac.uk
}

\begin{abstract}
This paper is concerned with the boundedness, exponential stability and almost sure asymptotic stability of stochastic functional differential equations (SFDEs) with Markovian switching. The key technique used is the method of multiple Lyapunov functions. We use two auxiliary functions to dominate the corresponding different Lyapunov function in different mode while the diffusion operator in different model is controlled by other multiple auxiliary functions. Our conditions on the diffusion operator are weaker than those in the related existing works.
\end{abstract}

Keywords and phrases: stochastic functional differential equations; Markovian switching; asymptotic stability; boundedness; generalized Itô's formula.

\section{Introduction}

Dynamic systems in many branches of science and industry do not only depend on the present states but also the past states. Stochastic functional differential equations (SFDEs), including stochastic delay differential equations (SDDEs), are often used to model these systems. The stability theory of SFDEs has received lots of attentions over past twenty years. A large number of stability results have been established (e.g. [2], [5], [6], [8], [17], [18]). In particular, many important works have been developed systematically in book [14]. On the other hand, these systems may experience abrupt changes in their structure and parameters caused by phenomena such as component failures or repairs, changing subsystem interconnections, and abrupt environmental disturbances. Continuous time Markovian chains have been used to

\footnotetext{
${ }^{*}$ Corresponding author.
} 
model these abrupt changes. Consequently, the SFDEs with Markovian switching, including SDDEs with Markovian switching, have been used to model many complicated systems.

In general, an $n$-dimensional SDDE with Markovian switching has the form

$$
\mathrm{d} \boldsymbol{x}(t)=\boldsymbol{f}_{1}(\boldsymbol{x}(t), \boldsymbol{x}(t-\tau), t, r(t)) \mathrm{d} t+\mathbf{g}_{1}(\boldsymbol{x}(t), \boldsymbol{x}(t-\tau), t, r(t)) \mathrm{d} B(t)
$$

on $t \geq 0$, where $\tau>0, r(t)$ is a right-continuous Markovian chain with a finite state space $S=\{1,2, \ldots, N\}$ and $B(t)$ is an $m$-dimensional Brownian motion. In the system, $\boldsymbol{x}(t)$ stands for the state while $r(t)$ for the mode. The stability problem of SDDEs with Markovian switching under different settings has been discussed, for example, in [10]-[13], [20]. Recently, $\mathrm{Hu}$, Mao and Shen [3] established some new generalized theorems on the asymptotic stability and boundedness of SDDEs with Markovian switching using different types of Lyapunov functions (e.g. polynomials with different degrees) for different modes. More precisely, they assumed that there exist functions $V \in C^{2,1}\left(R^{n} \times R_{+} \times S ; R_{+}\right), U_{1}, U_{2} \in C\left(R^{n} \times[-\tau, \infty) ; R_{+}\right)$ such that

$$
\begin{gathered}
\liminf _{|x| \rightarrow \infty, 0 \leq t<\infty} U_{1}(x, t)=\infty, \\
U_{1}(x, t) \leq V(x, t, i) \leq U_{2}(x, t), \\
\mathscr{L} V(x, y, t, i) \leq c_{1}-c_{2} U_{2}(x, t)+c_{3} U_{2}(y, t-\tau)
\end{gathered}
$$

for all $(x, y, t, i) \in R^{n} \times R^{n} \times R_{+} \times S$, where $c_{j} \geq 0, j=1,2,3$, with $c_{2}>c_{3}$.

It is noted that the future state of system (1.1) is not only dependent on the present state $\boldsymbol{x}(t)$ but also the past state $\boldsymbol{x}(t-\tau)$. However, there are many practical systems whose future state depends on the state over the whole time interval $[t-\tau, t]$ rather than at times $t-\tau$ and $t$. SFDEs with Markovian switching have therefore been developed to describe such systems. Generally speaking, an $n$-dimensional SFDE with Markovian switching has the form

$$
\mathrm{d} \boldsymbol{x}(t)=\boldsymbol{f}\left(\boldsymbol{x}_{t}, t, r(t)\right) \mathrm{d} t+\mathbf{g}\left(\boldsymbol{x}_{t}, t, r(t)\right) \mathrm{d} B(t),
$$

on $t \geq 0$, where $\boldsymbol{x}_{t}=\{\boldsymbol{x}(t+u): u \in[-\tau, 0]\}$. In particular, if define $\boldsymbol{f}\left(\boldsymbol{x}_{t}, t, r(t)\right)=$ $\boldsymbol{f}_{1}(\boldsymbol{x}(t), \boldsymbol{x}(t-\tau), t, r(t))$ and $\mathbf{g}\left(\boldsymbol{x}_{t}, t, r(t)\right)=\mathbf{g}_{1}(\boldsymbol{x}(t), \boldsymbol{x}(t-\tau), t, r(t))$, the SFDE (1.3) becomes the SDDE (1.1). For SFDEs with Markovian switching (1.3), Mao [9] investigated the existence, uniqueness and the Razumikhin-type theorem on exponential stability of the global solution (also see Chapter 8 in book [13]); Peng and Zhang [15] discussed some new Razumikhin-type theorems on pth moment stability; $\mathrm{Hu}$ and $\mathrm{Wu}$ [4] examined asymptotic boundedness of the global solution.

The aims of this paper are to examine the existence and uniqueness of the global solution of the SFDE (1.3) and to establish more general results on the asymptotic stability and boundedness of the solution under much weaker conditions than those imposed in the references mentioned above. To do so, we will use the idea in [3] to apply different types of Lyapunov 
functions for different modes of the underlying SFDE. It is observed that the diffusion operators (1.2) in all modes, for the $\operatorname{SDDE}(1.1)$, are controlled by the values of $U_{2}(\boldsymbol{x}(s), s)$ at times $s=t-\tau$ and $s=t$. When dealing with the SFDE (1.3), it is natural that the diffusion operators in all modes will be influenced by the historical states on the whole interval $[t-\tau, t]$. In this paper, we will use multiple auxiliary functions to describe this complicated situation. More precisely speaking, the diffusion operators will be controlled by multiple auxiliary functions $W_{j}(j=1,2, \cdots, L)$ with constant coefficients $\alpha_{j}, \beta_{j}(j=1,2, \cdots, L)$ (see (3.3) in Assumptions 3.2 of Section 3) or time-varying coefficients $b_{j}(t)(j=1,2, \cdots, L)$ (see (4.3) in Assumptions 4.1 of Section 4). As far as we know, it is first time that such general conditions are imposed to study the asymptotic boundedness and stability of SFDEs with Markovian switching.

This paper does not only present more general results on the boundedness and exponential stability of SFDEs with Markovian switching using the multiple auxiliary functions with constant coefficients but also discuss the almost sure asymptotic stability. Our results imply Theorem 3.1 in [3] and Theorem 8.4 in [13]. This paper also gives some further new results for SFDEs with Markovian switching using multiple auxiliary functions with time-varying coefficients.

The rest of this paper is organized as follows. In Section 2, we give some necessary notations. In Section 3, we discuss the asymptotic stability and boundedness of SFDEs with Markovian switching. Some further new results for SFDEs with Markovian switching using multiple auxiliary functions with time-varying coefficients are given in Section 4. We will finally conclude our paper in Section 5 .

\section{Preliminaries}

Throughout this paper, let $\left(\Omega, \mathscr{F},\left\{\mathscr{F}_{t}\right\}_{t \geq 0}, \mathbb{P}\right)$ be a complete probability space with a filtration $\left\{\mathscr{F}_{t}\right\}_{t \geq 0}$ satisfying the usual conditions (i.e., it is increasing and right continuous while $\mathscr{F}_{0}$ contains all $\mathbb{P}$-null sets). If $A$ is a matrix or vector, its transpose is denoted by $A^{T}$, and the trace norm of matrix $A$ is denoted by $|A|=\sqrt{\operatorname{trace}\left(A^{T} A\right)}$. Let $B(t)$ be an $m$ dimensional Brownian motion defined on the probability space. Let $\tau>0$ and $C\left([-\tau, 0] ; R^{n}\right)$ denote the family of all continuous $R^{n}$-valued functions $\varphi$ on $[-\tau, 0]$ with the norm $\|\varphi\|=$ $\sup _{-\tau \leq \theta \leq 0}|\varphi(\theta)|$. Let $C=C_{\mathscr{F}_{0}}^{b}\left([-\tau, 0] ; R^{n}\right)$ be the family of all bounded, $\mathscr{F}_{0}$-measurable, $C\left([-\tau, 0] ; R^{n}\right)$-valued, $\mathscr{F}_{t}$-adapted stochastic processes. Let $\eta_{j}$ be probability measures on $[-\tau, 0]$, which satisfy $\int_{-\tau}^{0} d \eta_{j}(\theta)=1(j=1,2, \cdots, L)$. Let $L^{1}\left(R_{+} ; R_{+}\right)$be the family of all Borel measurable functions $\xi: R_{+} \rightarrow R_{+}$such that $\int_{0}^{\infty} \xi(t) \mathrm{d} t<\infty$, and $\Psi\left(R_{+} ; R_{+}\right)$the family of all continuous functions $\psi: R_{+} \rightarrow R_{+}$such that $\liminf _{t \rightarrow \infty} \int_{t}^{t+\epsilon} \psi(t) \mathrm{d} t>0$, for any $\epsilon>0$. A continuous function $Q: R^{n} \rightarrow R_{+}$is said to be positive definite if $Q(\boldsymbol{x})=0$ iff $\boldsymbol{x}=0 . \boldsymbol{x}(t)$ is a continuous $R$-valued stochastic process on $t \in[-\tau, \infty)$. We assume 
$\boldsymbol{x}_{t}=\{\boldsymbol{x}(t+\theta):-\tau \leqslant \theta \leqslant 0\}$ for all $t \geq 0$, which is regarded as a $C\left([-\tau, 0] ; R^{n}\right)$-valued stochastic process.

Let $r(t), t \geq 0$, be a right-continuous Markovian chain on the probability space taking values in a finite state space $S=\{1,2, \ldots, N\}$, with generator $\Gamma=\left(\gamma_{i j}\right)_{N \times N}$ given by

$$
P\{r(t+\Delta)=j \mid r(t)=i\}=\left\{\begin{array}{l}
\gamma_{i j} \Delta+o(\Delta) \\
1+\gamma_{i i} \Delta+o(\Delta) \quad \text { if } \quad i \neq j, \quad i=j,
\end{array}\right.
$$

where $\Delta>0, \gamma_{i j}$ is the transition rate from $i$ to $j$, if $i \neq j$, while $\gamma_{i i}=-\sum_{j \neq i} \gamma_{i j}$.

Assume that Markovian chain $r(t)$ is independent of Brownian motion $B(t)$. It is well known that almost every sample path of $r(t)$ is a right-continuous step function.

It is useful to recall that a continuous-time Markovian chain $r(t)$ with generator $\Gamma=$ $\left(\gamma_{i j}\right)_{N \times N}$ can be represented as a stochastic integral with respect to a Poisson random measure

$$
\mathrm{d} r(t)=\int_{R} \bar{h}(r(t-), y) \nu(\mathrm{d} t, \mathrm{~d} y), \quad t \geq 0
$$

with initial value $r(0)=i_{0} \in S$, where $\nu(\mathrm{d} t, \mathrm{~d} y)$ is a Poisson random measure with intensity $\mathrm{d} t \times m(\mathrm{~d} y)$ in which $m$ is the Lebesgue measure on $R$, while the explicit definition of $\bar{h}$ : $S \times R \rightarrow R$ can be found in [1].

Consider an n-dimensional SFDE with Markovian switching of the form

$$
\mathrm{d} \boldsymbol{x}(t)=\boldsymbol{f}\left(\boldsymbol{x}_{t}, t, r(t)\right) \mathrm{d} t+\mathbf{g}\left(\boldsymbol{x}_{t}, t, r(t)\right) \mathrm{d} B(t),
$$

on $t \geq 0$ with initial data $\{\boldsymbol{x}(\theta):-\tau \leq \theta \leq 0\}=\zeta \in C_{\mathscr{F}_{0}}^{b}\left([-\tau, 0] ; R^{n}\right), i_{0} \in S$, where

$$
f: C\left([-\tau, 0] ; R^{n}\right) \times R_{+} \times S \rightarrow R^{n}, \quad \mathbf{g}: C\left([-\tau, 0] ; R^{n}\right) \times R_{+} \times S \rightarrow R^{n \times m} .
$$

Let $C\left(R^{n} \times[-\tau, \infty) ; R_{+}\right)$denote the family of all continuous functions from $R^{n} \times[-\tau,+\infty)$ to $R_{+}$, and $C^{2,1}\left(R^{n} \times[-\tau,+\infty) \times S ; R_{+}\right)$the family of all continuous nonnegative functions $V(x, t, i)$ on $R^{n} \times[-\tau,+\infty) \times S$ which are continuously twice differentiable in $x$ and once differentiable in $t$. For each $V \in C^{2,1}\left(R^{n} \times[-\tau,+\infty) \times S ; R_{+}\right)$, denote an operator $\mathscr{L} V$ from $C\left([-\tau, 0] ; R^{n}\right) \times R_{+} \times S$ to $R$ by

$$
\begin{aligned}
\mathscr{L} V(\boldsymbol{\varphi}, t, i)= & \sum_{j=1}^{N} \gamma_{i j} V(\boldsymbol{\varphi}(0), t, j)+V_{t}(\boldsymbol{\varphi}(0), t, i)+V_{x}(\boldsymbol{\varphi}(0), t, i) \boldsymbol{f}(\boldsymbol{\varphi}, t, i) \\
& +\frac{1}{2} \operatorname{trace}\left[\mathbf{g}^{T}(\boldsymbol{\varphi}, t, i) V_{x x}(\boldsymbol{\varphi}(0), t, i) \mathbf{g}(\boldsymbol{\varphi}, t, i)\right],
\end{aligned}
$$

where

$V_{t}(\boldsymbol{x}, t, i)=\frac{\partial V(\boldsymbol{x}, t, i)}{\partial t}, V_{x x}(\boldsymbol{x}, t, i)=\left(\frac{\partial^{2} V(\boldsymbol{x}, t, i)}{\partial x_{i} x_{j}}\right)_{n \times n}, V_{x}(\boldsymbol{x}, t, i)=\left(\frac{\partial V(\boldsymbol{x}, t, i)}{\partial x_{1}}, \cdots, \frac{\partial V(\boldsymbol{x}, t, i)}{\partial x_{n}}\right)$. 
For the convenience of readers we cite the generalized Itô's formula (see [13]): if $V \in$ $C^{2,1}\left(R^{n} \times[-\tau,+\infty) \times S ; R_{+}\right)$, then for any $t \geq 0$,

$$
\begin{aligned}
V(\boldsymbol{x}(t), t, r(t))= & V(\boldsymbol{x}(0), 0, r(0))+\int_{0}^{t} \mathscr{L} V\left(\boldsymbol{x}_{s}, s, r(s)\right) \mathrm{d} s+\int_{0}^{t} V_{x}(\boldsymbol{x}(s), s, r(s)) \mathbf{g}\left(\boldsymbol{x}_{s}, s, r(s)\right) \mathrm{d} B(s) \\
& +\int_{0}^{t} \int_{R} V\left(\boldsymbol{x}(s), s, i_{0}+\bar{h}(r(s-), l)\right)-V(\boldsymbol{x}(s), s, r(s)) \mu(\mathrm{d} s, \mathrm{~d} l),
\end{aligned}
$$

where $\mu(\mathrm{d} s, \mathrm{~d} l)=\nu(\mathrm{d} s, \mathrm{~d} l)-m(\mathrm{~d} l)$ is a martingale measure. Please note that this martingale measure is related to the Markov chain but not the Brownian motion (which forms another independent martingale in the formula above). The key benefit of this formula will allow us to apply the non-negative semi-martingale convergence theorem (see [7]) cited below as a lemma.

Lemma 2.1. Let $A_{1}(t)$ and $A_{2}(t)$ be two continuous adapted increasing processes on $t \geq 0$ with $A_{1}(0)=A_{2}(0)=0$ a.s., $M(t)$ a real-valued continuous local martingale with $M(0)=0$ a.s., $\zeta$ a nonnegative $\mathscr{F}_{0}$-measurable random variable such that $E \zeta<\infty$. Define $X(t)=$ $\zeta+A_{1}(t)-A_{2}(t)+M(t)$ for $t \geq 0$. If $X(t)$ is nonnegative, then

$$
\left\{\lim _{t \rightarrow \infty} A_{1}(t)<\infty\right\} \subset\left\{\lim _{t \rightarrow \infty} X(t)<\infty\right\} \cap\left\{\lim _{t \rightarrow \infty} A_{2}(t)<\infty\right\} \quad \text { a.s. }
$$

where $C \subset D$ a.s. means $\mathbb{P}\left(C \cap D^{c}\right)=0$. In particular, if $\lim _{t \rightarrow \infty} A_{1}(t)<\infty$ a.s., then, with probability one,

$$
\lim _{t \rightarrow \infty} X(t)<\infty, \quad \lim _{t \rightarrow \infty} A_{2}(t)<\infty, \quad-\infty<\lim _{t \rightarrow \infty} M(t)<\infty .
$$

For the stability purpose, we furthermore assume that $\boldsymbol{f}(0, t, i)=0$ and $\mathbf{g}(0, t, i)=0$ for all $t \in R_{+}, i \in S$, so that system (2.1) admits a trivial solution $\boldsymbol{x}(t)=0$.

\section{General Asymptotic Stability and Boundedness of SFDEs with Markovian switching}

In this section, we give the results for the existence and uniqueness, general asymptotic stability and general boundedness of the global solutions of SFDEs with Markovian switching using multiple auxiliary functions with constant coefficients.

To get our main results, we firstly put forward the following assumptions.

Assumption 3.1. Both $\boldsymbol{f}$ and $\mathbf{g}$ satisfy the local Lipschitz condition. That is, for each $k=1,2, \cdots$, there is a $\mathrm{c}_{k}>0$ such that

$$
|\boldsymbol{f}(\boldsymbol{\varphi}, t, i)-\boldsymbol{f}(\boldsymbol{\psi}, t, i)| \vee|\mathbf{g}(\boldsymbol{\varphi}, t, i)-\mathbf{g}(\boldsymbol{\psi}, t, i)| \leq \mathrm{c}_{k}\|\boldsymbol{\varphi}-\boldsymbol{\psi}\|,
$$

for all $t \in R_{+}, i \in S$ and $\boldsymbol{\varphi}, \boldsymbol{\psi} \in C\left([-\tau, 0] ; R^{n}\right)$ with $\|\boldsymbol{\varphi}\| \vee\|\boldsymbol{\psi}\| \leq k$. 
Assumption 3.2. There exist functions $V \in C^{2,1}\left(R^{n} \times[-\tau, \infty) \times S ; R_{+}\right), W_{0}, W_{j} \in C\left(R^{n} \times\right.$ $\left.[-\tau, \infty) ; R_{+}\right)$and probability measures $\eta_{j}$ on $[-\tau, 0]$ satisfying $\int_{-\tau}^{0} d \eta_{j}(\theta)=1$, as well as nonnegative numbers $\alpha_{0}, \alpha_{j}, \beta_{j}$ satisfying $\alpha_{j}>\beta_{j}(j=1,2, \cdots, L)$, such that

$$
\begin{gathered}
\liminf _{|x| \rightarrow \infty, 0 \leq t<\infty} W_{0}(x, t)=\infty, \\
W_{0}(x, t) \leq V(x, t, i) \leq W_{1}(x, t), \quad \forall(x, t, i) \in C\left([-\tau, 0] ; R^{n}\right) \times R_{+} \times S, \\
\mathscr{L} V(\boldsymbol{\varphi}, t, i) \leq \alpha_{0}+\sum_{j=1}^{L}\left[-\alpha_{j} W_{j}(\varphi(0), t)+\beta_{j} \int_{-\tau}^{0} W_{j}(\varphi(\theta), t+\theta) d \eta_{j}(\theta)\right],
\end{gathered}
$$

for all $i \in S$ and $\varphi \in C\left([-\tau, 0] ; R^{n}\right), t \in R_{+}$.

Remark 1. Assumption 3.2 is a generalization of Assumption 2.2 of [3] (condition (1.2) in this paper). The generalization mainly shows in the following two aspects:

1) Assumption 3.2 introduces multiple auxiliary functions $W_{j}(j=0,1, \cdots, L)$ instead of two functions in Assumption 2.2 of [3];

2) When the probability measure $\eta_{1}(\theta)$ is a point probability measure at $-\tau$, Assumption 3.2 with $\alpha_{j}=\beta_{j}=0(j=2,3, \cdots, L)$ reduces to Assumption 2.2 of [3].

Since the coefficients of $W_{j}(j=1, \cdots, L)$ in $(3.3)$ are constants, it is said to be the case of multiple auxiliary functions with constant coefficients for convenience. Now, we state our main results of SFDEs with Markovian switching in this case.

Theorem 3.1. Under Assumptions 3.1 and 3.2, we have the following assertions:

1) For any given initial data $\boldsymbol{\zeta} \in C$ and $i_{0} \in S$, there is a unique global solution $\boldsymbol{x}\left(t, \boldsymbol{\zeta}, i_{0}\right)$ of system (2.1) on $t \geq-\tau$, which satisfies the following moment properties

$$
\begin{gathered}
\limsup _{t \rightarrow \infty} E W_{0}\left(\boldsymbol{x}\left(t, \boldsymbol{\zeta}, i_{0}\right), t\right) \leq \frac{\alpha_{0}}{\varepsilon_{0}} \\
\limsup _{t \rightarrow \infty} \frac{1}{t} \int_{0}^{t} E W_{j}\left(\boldsymbol{x}\left(s, \boldsymbol{\zeta}, i_{0}\right), s\right) \mathrm{d} s \leq \frac{\alpha_{0}}{\alpha_{j}-\beta_{j}}, \quad j=1,2, \cdots, L
\end{gathered}
$$

where $\varepsilon_{0}=\sup \left\{\varepsilon>0: \varepsilon+\beta_{1} e^{\varepsilon \tau}-\alpha_{1} \leq 0, \varepsilon \leq \frac{1}{\tau} \min _{j \geq 2}\left\{\log \frac{\alpha_{j}}{\beta_{j}}\right\}\right\}$.

2) Moreover, if $\alpha_{0}=0$, the global solution $\boldsymbol{x}\left(t, \boldsymbol{\zeta}, i_{0}\right)$ has the following moment properties

$$
\begin{gathered}
\limsup _{t \rightarrow \infty} \frac{1}{t} \log \left(E W_{0}\left(\boldsymbol{x}\left(t, \boldsymbol{\zeta}, i_{0}\right), t\right)\right) \leq-\varepsilon_{0}, \\
\int_{0}^{\infty} E W_{j}\left(\boldsymbol{x}\left(s, \boldsymbol{\zeta}, i_{0}\right), s\right) \mathrm{d} s<\infty, \quad j=1,2, \cdots, L
\end{gathered}
$$


and the following sample properties

$$
\begin{gathered}
\sup _{-\tau \leq t<\infty} \boldsymbol{x}\left(t, \boldsymbol{\zeta}, i_{0}\right)<\infty, \quad \text { a.s. } \\
\limsup _{t \rightarrow \infty} \frac{1}{t} \log \left(W_{0}\left(\boldsymbol{x}\left(t, \boldsymbol{\zeta}, i_{0}\right), t\right)\right) \leq-\varepsilon_{0}, \quad \text { a.s. } \\
\int_{0}^{\infty} W_{j}\left(\boldsymbol{x}\left(s, \boldsymbol{\zeta}, i_{0}\right), s\right) \mathrm{d} s<\infty . \quad \text { a.s. } \quad j=1,2, \cdots, L
\end{gathered}
$$

3) In the case $\alpha_{0}=0$, and there furthermore exists a continuous positive definite function $Q \in C\left(R^{n} ; R_{+}\right)$such that $Q(\boldsymbol{x}) \leq \sum_{j=1}^{L} W_{j}(\boldsymbol{x}, t)$ for all $(\boldsymbol{x}, t) \in R^{n} \times[-\tau, \infty)$, then

$$
\lim _{t \rightarrow \infty} \boldsymbol{x}\left(t, \boldsymbol{\zeta}, i_{0}\right)=0 . \quad \text { a.s. }
$$

Proof. 1) By Assumption 3.1, for any given initial data $\zeta \in C$ and $i_{0} \in S$, there is a unique maximal local solution $\boldsymbol{x}\left(t, \boldsymbol{\zeta}, i_{0}\right)$ on $t \in\left[-\tau, \sigma_{\infty}\right)$, where $\sigma_{\infty}$ is the explosion time. Let $k_{0}>0$ be sufficiently large satisfying $\|\zeta\|<k_{0}$. For each integer $k \geq k_{0}$, define the stopping time

$$
\tau_{k}=\inf \left\{t \in\left[0, \sigma_{\infty}\right):|x(t)| \geq k\right\} .
$$

Obviously, $\tau_{k}$ is increasing as $k \rightarrow \infty$. Let $\tau_{\infty}=\lim _{t \rightarrow \infty} \tau_{k}$, so $\tau_{\infty} \leq \sigma_{\infty}$ a.s.. If we can obtain that $\tau_{\infty}=\infty$ a.s., then $\sigma_{\infty}=\infty$ a.s.. For the sake of simplicity, write $\boldsymbol{x}(t)=\boldsymbol{x}\left(t, \boldsymbol{\zeta}, i_{0}\right)$. Using the generalized Itô's formula and then taking the expectation, we have

$$
\begin{aligned}
& E V\left(\boldsymbol{x}\left(t \wedge \tau_{k}\right), t \wedge \tau_{k}, r\left(t \wedge \tau_{k}\right)\right)=E V(\boldsymbol{x}(0), 0, r(0))+E \int_{0}^{t \wedge \tau_{k}} \mathscr{L} V\left(\boldsymbol{x}_{s}, s, r(s)\right) \mathrm{d} s \\
& \quad \leq E V(\boldsymbol{x}(0), 0, r(0))+E \int_{0}^{t \wedge \tau_{k}}\left[\alpha_{0}+\sum_{j=1}^{L}\left[-\alpha_{j} W_{j}(\boldsymbol{x}(s), s)+\beta_{j} \int_{-\tau}^{0} W_{j}(\boldsymbol{x}(s+\theta), s+\theta) d \eta_{j}(\theta)\right]\right] \mathrm{d} s \\
& \quad \leq E V(\boldsymbol{x}(0), 0, r(0))+E \int_{0}^{t \wedge \tau_{k}}\left[\alpha_{0}+\sum_{j=1}^{L}\left[-\left(\alpha_{j}-\beta_{j}\right) W_{j}(\boldsymbol{x}(s), s)+\beta_{j} J_{j}\right]\right] \mathrm{d} s
\end{aligned}
$$

where $J_{j}=\int_{-\tau}^{0} W_{j}(\boldsymbol{x}(s+\theta), s+\theta) \mathrm{d} \eta_{j}(\theta)-W_{j}(\boldsymbol{x}(s), s), j=1,2, \cdots, L$.

By virtue of the fact that

$$
\begin{aligned}
\int_{0}^{t \wedge \tau_{k}} J_{j} \mathrm{~d} s & =\int_{0}^{t \wedge \tau_{k}}\left[\int_{-\tau}^{0} W_{j}(\boldsymbol{x}(s+\theta), s+\theta) \mathrm{d} \eta_{j}(\theta)-W_{j}(\boldsymbol{x}(s), s)\right] \mathrm{d} s \\
& =\int_{-\tau}^{0} \int_{\theta}^{\left(t \wedge \tau_{k}\right)+\theta} W_{j}(\boldsymbol{x}(s), s) \mathrm{d} s \mathrm{~d} \eta_{j}(\theta)-\int_{0}^{t \wedge \tau_{k}} W_{j}(\boldsymbol{x}(s), s) \mathrm{d} s \\
& \leq \int_{-\tau}^{0} \int_{-\tau}^{t \wedge \tau_{k}} W_{j}(\boldsymbol{x}(s), s) \mathrm{d} s \mathrm{~d} \eta_{j}(\theta)-\int_{0}^{t \wedge \tau_{k}} W_{j}(\boldsymbol{x}(s), s) \mathrm{d} s \\
& \leq \int_{-\tau}^{t \wedge \tau_{k}} W_{j}(\boldsymbol{x}(s), s) \mathrm{d} s-\int_{0}^{t \wedge \tau_{k}} W_{j}(\boldsymbol{x}(s), s) \mathrm{d} s
\end{aligned}
$$




$$
\leq \int_{-\tau}^{0} W_{j}(\boldsymbol{x}(s), s) \mathrm{d} s
$$

for $j=1,2, \cdots, L,(3.12)$ can be rewritten as

$$
\begin{aligned}
E V\left(\boldsymbol{x}\left(t \wedge \tau_{k}\right), t \wedge \tau_{k}, r\left(t \wedge \tau_{k}\right)\right) \leq & a_{0} E\left(t \wedge \tau_{k}\right)-\sum_{j=1}^{L}\left(\alpha_{j}-\beta_{j}\right) E \int_{0}^{t \wedge \tau_{k}} W_{j}(\boldsymbol{x}(s), s) \mathrm{d} s \\
& +E V(\boldsymbol{x}(0), 0, r(0))+\sum_{j=1}^{L} \beta_{j} E \int_{-\tau}^{0} W_{j}(\boldsymbol{x}(s), s) \mathrm{d} s \\
\leq & c_{0}+a_{0} t-\sum_{j=1}^{L}\left(\alpha_{j}-\beta_{j}\right) E \int_{0}^{t \wedge \tau_{k}} W_{j}(\boldsymbol{x}(s), s) \mathrm{d} s,(3 .
\end{aligned}
$$

where $c_{0}=E V(\boldsymbol{x}(0), 0, r(0))+\sum_{j=1}^{L} \beta_{j} E \int_{-\tau}^{0} W_{j}(\boldsymbol{x}(s), s) \mathrm{d} s$.

From condition $(3.2)$ and $\alpha_{j}>\beta_{j}(j=1,2, \cdots, L)$, we get that

$$
E W_{0}\left(\boldsymbol{x}\left(t \wedge \tau_{k}\right), t \wedge \tau_{k}\right) \leq c_{0}+a_{0} t
$$

Let $\nu_{k}=\inf _{|x| \geq k, 0 \leq t<\infty} W_{0}(\boldsymbol{x}(t), t)$, for $k \geq k_{0}$, then we have that

$$
\left.E W_{0}\left(\boldsymbol{x}\left(t \wedge \tau_{k}\right), t \wedge \tau_{k}\right) \geq E\left(W_{0}\left(\tau_{k}\right), \tau_{k}\right) I_{\left\{\tau_{k} \leq t\right\}}\right) \geq \nu_{k} P\left(\tau_{k} \leq t\right) .
$$

Letting $k \rightarrow \infty$ in the inequality above, we have that

$$
P\left(\tau_{\infty} \leq t\right)=\lim _{k \rightarrow \infty} P\left(\tau_{k} \leq t\right) \leq \lim _{k \rightarrow \infty} \frac{E W_{0}\left(\boldsymbol{x}\left(t \wedge \tau_{k}\right), t \wedge \tau_{k}\right)}{\nu_{k}}=\lim _{k \rightarrow \infty} \frac{c_{0}+a_{0} t}{\nu_{k}}=0 .
$$

Since $t>0$ is arbitrary, we must have that $\tau_{\infty}=\infty$ a.s.. Hence $\sigma_{\infty}=\infty$ a.s., which implies that the unique maximal local solution $x(t)$ on $t \in\left[-\tau, \sigma_{\infty}\right)$ becomes the unique global solution on $t \in[-\tau, \infty)$.

Using the generalized Itô's formula to $e^{\varepsilon t} V(\boldsymbol{x}(t), t, r(t)), \varepsilon \in\left(0, \varepsilon_{0}\right]$, and from condition (3.2), we get that, for any $t \geq 0$,

$$
\begin{aligned}
e^{\varepsilon\left(t \wedge \tau_{k}\right)} & V\left(\boldsymbol{x}\left(t \wedge \tau_{k}\right), t \wedge \tau_{k}, r\left(t \wedge \tau_{k}\right)\right) \\
= & V(\boldsymbol{x}(0), 0, r(0))+\int_{0}^{t \wedge \tau_{k}} e^{\varepsilon s}\left(\varepsilon V(\boldsymbol{x}(s), s, r(s))+\mathscr{L} V\left(\boldsymbol{x}_{s}, s, r(s)\right)\right) \mathrm{d} s+M\left(t \wedge \tau_{k}\right) \\
\leq & W_{1}(\boldsymbol{x}(0), 0)+\int_{0}^{t \wedge \tau_{k}} e^{\varepsilon s}\left[\alpha_{0}-\left(\alpha_{1}-\varepsilon\right) W_{1}(\boldsymbol{x}(s), s)+\beta_{1} \int_{-\tau}^{0} W_{1}(\boldsymbol{x}(s+\theta), s+\theta) d \eta_{1}(\theta)\right. \\
& \left.+\sum_{j=2}^{L}\left[-\alpha_{j} W_{j}(\boldsymbol{x}(s), s)+\beta_{j} \int_{-\tau}^{0} W_{j}(\boldsymbol{x}(s+\theta), s+\theta) d \eta_{j}(\theta)\right]\right] \mathrm{d} s+M\left(t \wedge \tau_{k}\right) \\
\leq & W_{1}(\boldsymbol{x}(0), 0)+\int_{0}^{t \wedge \tau_{k}} e^{\varepsilon s}\left[\alpha_{0}-\left(\alpha_{1}-\varepsilon-\beta_{1} e^{\varepsilon \tau}\right) W_{1}(\boldsymbol{x}(s), s)+\beta_{1} J_{1}^{\prime}\right.
\end{aligned}
$$




$$
\left.+\sum_{j=2}^{L}\left[-\left(\alpha_{j}-\beta_{j} e^{\varepsilon \tau}\right) W_{j}(\boldsymbol{x}(s), s)+\beta_{j} J_{j}^{\prime}\right]\right] \mathrm{d} s+M\left(t \wedge \tau_{k}\right),
$$

where $M\left(t \wedge \tau_{k}\right)$ is a local martingale with the initial value $M(0)=0, J_{j}^{\prime}=\int_{-\tau}^{0} W_{j}(\boldsymbol{x}(s+$ $\theta), s+\theta) \mathrm{d} \eta_{j}(\theta)-e^{\varepsilon \tau} W_{j}(\boldsymbol{x}(s), s), j=1,2, \cdots L$.

By virtue of the fact that

$$
\begin{aligned}
\int_{0}^{t \wedge \tau_{k}} e^{\varepsilon s} J_{j}^{\prime} \mathrm{d} s & =\int_{0}^{t \wedge \tau_{k}} e^{\varepsilon s}\left[\int_{-\tau}^{0} W_{j}(\boldsymbol{x}(s+\theta), s+\theta) \mathrm{d} \eta_{j}(\theta)-e^{\varepsilon \tau} W_{j}(\boldsymbol{x}(s), s)\right] \mathrm{d} s \\
& =\int_{-\tau}^{0} \int_{\theta}^{\left(t \wedge \tau_{k}\right)+\theta} e^{\varepsilon(s-\theta)} W_{j}(\boldsymbol{x}(s), s) \mathrm{d} s \mathrm{~d} \eta_{j}(\theta)-e^{\varepsilon \tau} \int_{0}^{t \wedge \tau_{k}} e^{\varepsilon s} W_{j}(\boldsymbol{x}(s), s) \mathrm{d} s \\
& \leq \int_{-\tau}^{0} \int_{-\tau}^{t \wedge \tau_{k}} e^{\varepsilon(s+\tau)} W_{j}(\boldsymbol{x}(s), s) \mathrm{d} s \mathrm{~d} \eta_{j}(\theta)-e^{\varepsilon \tau} \int_{0}^{t \wedge \tau_{k}} e^{\varepsilon s} W_{j}(\boldsymbol{x}(s), s) \mathrm{d} s \\
& \leq e^{\varepsilon \tau} \int_{-\tau}^{t \wedge \tau_{k}} e^{\varepsilon s} W_{j}(\boldsymbol{x}(s), s) \mathrm{d} s-e^{\varepsilon \tau} \int_{0}^{t \wedge \tau_{k}} e^{\varepsilon s} W_{j}(\boldsymbol{x}(s), s) \mathrm{d} s \\
& \leq e^{\varepsilon \tau} \int_{-\tau}^{0} W_{j}(\boldsymbol{x}(s), s) \mathrm{d} s
\end{aligned}
$$

for $j=1,2, \cdots, L,(3.16)$ can be rewritten as

$$
\begin{aligned}
& e^{\varepsilon\left(t \wedge \tau_{k}\right)} V\left(\boldsymbol{x}\left(t \wedge \tau_{k}\right), t \wedge \tau_{k}, r\left(t \wedge \tau_{k}\right)\right) \\
& \leq W_{1}(\boldsymbol{x}(0), 0)+\frac{\alpha_{0}}{\varepsilon}\left(e^{\varepsilon\left(t \wedge \tau_{k}\right)}-1\right)-\left(\alpha_{1}-\varepsilon-\beta_{1} e^{\varepsilon \tau}\right) \int_{0}^{t \wedge \tau_{k}} e^{\varepsilon s} W_{1}(\boldsymbol{x}(s), s) \mathrm{d} s \\
&-\sum_{j=2}^{L}\left(\alpha_{j}-\beta_{j} e^{\varepsilon \tau}\right) \int_{0}^{t \wedge \tau_{k}} e^{\varepsilon s} W_{j}(\boldsymbol{x}(s), s) \mathrm{d} s+\sum_{j=1}^{L} \beta_{j} e^{\varepsilon \tau} \int_{-\tau}^{0} W_{j}(\boldsymbol{x}(s), s) \mathrm{d} s+M\left(t \wedge \tau_{k}\right) \\
& \leq c_{1}+\frac{\alpha_{0}}{\varepsilon} e^{\varepsilon\left(t \wedge \tau_{k}\right)}-\left(\alpha_{1}-\varepsilon-\beta_{1} e^{\varepsilon \tau}\right) \int_{0}^{t \wedge \tau_{k}} e^{\varepsilon s} W_{1}(\boldsymbol{x}(s), s) \mathrm{d} s \\
&-\sum_{j=2}^{L}\left(\alpha_{j}-\beta_{j} e^{\varepsilon \tau}\right) \int_{0}^{t \wedge \tau_{k}} e^{\varepsilon s} W_{j}(\boldsymbol{x}(s), s) \mathrm{d} s+M\left(t \wedge \tau_{k}\right),
\end{aligned}
$$

where $c_{1}=W_{1}(\boldsymbol{x}(0), 0)+\sum_{j=1}^{L} \beta_{j} e^{\varepsilon \tau} \int_{-\tau}^{0} W_{j}(\boldsymbol{x}(s), s) \mathrm{d} s$.

Letting $k \rightarrow \infty$ and then taking the expectation on both side of (3.18), we get

$$
E\left(e^{\varepsilon t} W_{0}(\boldsymbol{x}(t), t)\right) \leq c_{1}+\frac{\alpha_{0}}{\varepsilon} e^{\varepsilon t},
$$

from the definition of $\varepsilon_{0}$ and condition (3.2). So letting $\varepsilon \rightarrow \varepsilon_{0}$, we have the assertion (3.4).

From (3.14), letting $k \rightarrow \infty$, we get that

$$
\sum_{j=1}^{L}\left(\alpha_{j}-\beta_{j}\right) E \int_{0}^{t} W_{j}(\boldsymbol{x}(s), s) \mathrm{d} s \leq c_{0}+a_{0} t .
$$

Letting $t \rightarrow \infty$ and using the Fubini theorem, we have the assertion (3.5). 
2) Next, we prove the results in the case of $\alpha_{0}=0$. From (3.19) we get that

$$
E W_{0}(\boldsymbol{x}(t), t) \leq c_{1} e^{-\varepsilon t}
$$

So letting $\varepsilon \rightarrow \varepsilon_{0}$, we get the assertion (3.6).

Moreover, (3.20) becomes

$$
\sum_{j=1}^{L}\left(\alpha_{j}-\beta_{j}\right) E \int_{0}^{t} W_{j}(\boldsymbol{x}(s), s) \mathrm{d} s \leq c_{0} .
$$

So letting $t \rightarrow \infty$, we get the assertion (3.7).

In the same way as (3.16), we get that

$$
e^{\varepsilon t} W_{0}(\boldsymbol{x}(t), t) \leq c_{1}+M(t)
$$

where $c_{1}$ has been defined above. By virtue of Lemma 2.1, we obtain that

$$
\limsup _{t \rightarrow \infty} e^{\varepsilon t} W_{0}(\boldsymbol{x}(t), t)<\infty . \quad \text { a.s. }
$$

Hence, there exists a finite positive random variable $\zeta$ such that

$$
\sup _{0 \leq t<\infty} e^{\varepsilon t} W_{0}(\boldsymbol{x}(t), t)<\zeta . \quad \text { a.s. }
$$

So as $\varepsilon \rightarrow \varepsilon_{0}$, we claim the assertion (3.9).

Using the generalized Itô's formula to $V(\boldsymbol{x}(t), t, r(t))$, we obtain

$$
\begin{aligned}
& V(\boldsymbol{x}(t), t, r(t))=V(\boldsymbol{x}(0), 0, r(0))+\int_{0}^{t} \mathscr{L} V\left(\boldsymbol{x}_{s}, s, r(s)\right) \mathrm{d} s+M^{\prime}(t) \\
& \leq W_{1}(\boldsymbol{x}(0), 0)+\int_{0}^{t} \sum_{j=1}^{L}\left[-\alpha_{j} W_{j}(\boldsymbol{x}(s), s)+\beta_{j} \int_{-\tau}^{0} W_{j}(\boldsymbol{x}(s+\theta), s+\theta) d \eta_{j}(\theta)\right] \mathrm{d} s+M^{\prime}(t) \\
& \leq W_{1}(\boldsymbol{x}(0), 0)+\sum_{j=1}^{L} \beta_{j} \int_{-\tau}^{0} W_{j}(\boldsymbol{x}(s), s) \mathrm{d} s-\sum_{j=1}^{L}\left(\alpha_{j}-\beta_{j}\right) \int_{0}^{t} W_{j}(\boldsymbol{x}(s), s) \mathrm{d} s+M^{\prime}(t)
\end{aligned}
$$

where $M^{\prime}(t)$ is a local martingale with the initial value $M^{\prime}(0)=0$. Due to Lemma 2.1, we obtain the assertions $(3.10)$ and $\sup _{-\tau \leq t<\infty} W_{0}(\boldsymbol{x}(t), t)<\infty$, a.s.. From the radial unboundedness of $W_{0}(x, t)$ (see (3.1)), we directly get the assertion (3.8).

3) Finally, we prove the result (3.11). Because of the positive definiteness of $Q(\boldsymbol{x})$, the assertion (3.11) is equivalent to the following assertion

$$
\lim _{t \rightarrow \infty} Q(\boldsymbol{x}(t))=0 . \quad \text { a.s. }
$$

Further, the assertion (3.23) is equivalent to the following assertion

$$
\limsup _{t \rightarrow \infty} Q(\boldsymbol{x}(t))=0 . \quad \text { a.s. }
$$


Now we claim that the assertion (3.24) holds. If (3.24) is false, we must have that

$$
P\left\{\limsup _{t \rightarrow \infty} Q(\boldsymbol{x}(t))>0\right\}>0 .
$$

Then there exists a constant $\delta$ such that $P(A)>3 \delta$, where $A=\left\{\omega: \limsup _{t \rightarrow \infty} Q(\boldsymbol{x}(t))>2 \delta\right\}$. From the assertion (3.8), we can see that there exists a sufficiently large positive constant $h=h(\delta)$ such that $P\left(A_{1}\right)>1-\delta$, where $A_{1}=\left\{\omega: \sup _{-\tau \leq t<\infty}|\boldsymbol{x}(t)|<h\right\}$. So we easily obtain that $P\left(A \cap A_{1}\right)>2 \delta$.

Define a sequence of stopping times by

$$
\begin{aligned}
& \mu_{1}=\inf \{t \geq 0: Q(\boldsymbol{x}(t)) \geq 2 \delta\}, \\
& \mu_{2 l}=\inf \left\{t \geq \mu_{2 l-1}: Q(\boldsymbol{x}(t)) \leq \delta\right\}, \quad l=1,2, \ldots \\
& \mu_{2 l+1}=\inf \left\{t \geq \mu_{2 l}: Q(\boldsymbol{x}(t)) \geq 2 \delta\right\} . \quad l=1,2, \ldots
\end{aligned}
$$

Obviously, when $\omega \in A \cap A_{1}$, then $\mu_{l}<\infty, l=1,2, \ldots$, i.e.

$$
A \cap A_{1} \subset\left\{\mu_{l}<\infty, l=1,2, \ldots\right\} .
$$

From Assumptions 3.1, $\boldsymbol{f}(0, t, i)=0$ and $\mathbf{g}(0, t, i)=0$, we get that there exists a constant $H_{h}>0$ such that $|\boldsymbol{f}(\boldsymbol{\varphi}, t, i)| \vee|\mathbf{g}(\boldsymbol{\varphi}, t, i)| \leq H_{h}$, for all $t \in R_{+}, i \in S$ and $\varphi \in C\left([-\tau, 0] ; R^{n}\right)$ with $\|\varphi\| \leq h$. Using Hölder's inequality and Burkholder-Davis-Gundy inequality, we obtain that, for any $T_{1}>0$,

$$
\begin{aligned}
E\left(I_{A \cap A_{1}} \sup _{0 \leq t \leq T_{1}}\left|\boldsymbol{x}\left(\mu_{2 l-1}+t\right)-\boldsymbol{x}\left(\mu_{2 l-1}\right)\right|^{2}\right) & \leq 2 E\left(I_{A \cap A_{1}} \sup _{0 \leq t \leq T_{1}}\left|\int_{\mu_{2 l-1}}^{\mu_{2 l-1}+t} \boldsymbol{f}\left(\boldsymbol{x}_{s}, s, r(s)\right) \mathrm{d} s\right|^{2}\right) \\
& +2 E\left(I_{A \cap A_{1}} \sup _{0 \leq t \leq T_{1}}\left|\int_{\mu_{2 l-1}}^{\mu_{2 l-1}+t} \mathbf{g}\left(\boldsymbol{x}_{s}, s, r(s)\right) \mathrm{d} B(s)\right|^{2}\right) \\
\leq & 2 T_{1} H_{h}^{2}\left(T_{1}+4\right)
\end{aligned}
$$

Since $Q(\boldsymbol{x})$ is continuous in $R^{n}$, it is uniformly continuous in the closed ball $\bar{B}_{h}=\left\{\boldsymbol{x} \in R^{n}\right.$ : $|\boldsymbol{x}| \leq h\}$. Hence, we can choose a sufficiently small $\delta^{\prime}=\delta^{\prime}(\delta)>0$ such that $|Q(\boldsymbol{x})-Q(\boldsymbol{y})|<\delta$, when $|\boldsymbol{x}| \vee|\boldsymbol{y}| \leq h$ and $|\boldsymbol{x}-\boldsymbol{y}| \leq \delta^{\prime}$.

By using Chebyshev's inequality, we get that

$$
\begin{aligned}
P(A & \left.\cap A_{1} \cap\left\{\sup _{0 \leq t \leq T_{1}}\left|\boldsymbol{x}\left(\mu_{2 l-1}+t\right)-\boldsymbol{x}\left(\mu_{2 l-1}\right)\right|<\delta^{\prime}\right\}\right) \\
& =P\left(A \cap A_{1}\right)-E\left(I_{A \cap A_{1}} \sup _{0 \leq t \leq T_{1}}\left|\boldsymbol{x}\left(\mu_{2 l-1}+t\right)-\boldsymbol{x}\left(\mu_{2 l-1}\right)\right| \geq \delta^{\prime}\right) \geq 2 \delta-\frac{2 T_{1} H_{h}^{2}\left(T_{1}+4\right)}{\delta^{\prime 2}} .
\end{aligned}
$$

Noting that $\left\{\sup _{0 \leq t \leq T_{1}}\left|\boldsymbol{x}\left(\mu_{2 l-1}+t\right)-\boldsymbol{x}\left(\mu_{2 l-1}\right)\right| \leq \delta^{\prime}\right\} \subset\left\{\sup _{0 \leq t \leq T_{1}}\left|Q\left(\boldsymbol{x}\left(\mu_{2 l-1}+t\right)\right)-Q\left(\boldsymbol{x}\left(\mu_{2 l-1}\right)\right)\right|<\right.$ $\delta\} \subset\left\{\mu_{2 l}-\mu_{2 l-1} \geq T_{1}\right\}$, we choose a sufficiently small $T_{1}>0$ such that $\frac{2 T_{1} H_{h}^{2}\left(T_{1}+4\right)}{\delta^{\prime 2}}<\delta$. So we compute that $P\left(A \cap A_{1} \cap\left\{\sup _{0 \leq t \leq T_{1}}\left|\boldsymbol{x}\left(\mu_{2 l-1}+t\right)-\boldsymbol{x}\left(\mu_{2 l-1}\right)\right|<\delta^{\prime}\right\}\right)>\delta$. 
On the other hand, from the assertion (3.7) and the relation of $Q(\boldsymbol{x})$ and $\sum_{j=1}^{L} W_{j}(\boldsymbol{x}, t)$, we easily get that

$$
E \int_{0}^{\infty} Q(\boldsymbol{x}(s)) \mathrm{d} s \leq \sum_{j=1}^{L} E \int_{0}^{\infty} W_{j}\left(\boldsymbol{x}\left(s, \boldsymbol{\zeta}, i_{0}\right), s\right) \mathrm{d} s<\infty .
$$

For any $\omega \in A \cap A_{1} \cap\left\{\sup _{0 \leq t \leq T_{1}}\left|\boldsymbol{x}\left(\mu_{2 l-1}+t\right)-\boldsymbol{x}\left(\mu_{2 l-1}\right)\right|<\delta^{\prime}\right\}$, we have

$$
\begin{aligned}
E \int_{0}^{\infty} Q(\boldsymbol{x}(s)) \mathrm{d} s & \geq \sum_{l=1}^{\infty} E\left(I_{\left\{\mu_{l}<\infty\right\}} \int_{\mu_{2 l-1}}^{\mu_{2 l}} Q(\boldsymbol{x}(s)) \mathrm{d} s\right) \\
& \geq \delta \sum_{l=1}^{\infty} E\left(I_{A \cap A_{1} \cap\left\{\sup _{0 \leq t \leq T_{1}}\left|\boldsymbol{x}\left(\mu_{2 l-1}+t\right)-\boldsymbol{x}\left(\mu_{2 l-1}\right)\right|<\delta^{\prime}\right\}}\left(\mu_{2 l}-\mu_{2 l-1}\right)\right) \\
& \geq \delta T_{1} \sum_{l=1}^{\infty} E\left(I_{A \cap A_{1} \cap\left\{\sup _{0 \leq t \leq T_{1}}\left|\boldsymbol{x}\left(\mu_{2 l-1}+t\right)-\boldsymbol{x}\left(\mu_{2 l-1}\right)\right|<\delta^{\prime}\right\}}\right) \\
& \geq \delta T_{1} \sum_{l=1}^{\infty} \delta \\
& =\infty
\end{aligned}
$$

which implies a contradiction with (3.26). So we finish the proof of (3.11).

Remark 2. 1) The results obtained in Theorem 3.1 are in terms of functions $W_{j}(j=$ $0,1, \cdots, L)$, that are more general boundedness, general exponential stability and general $H_{\infty}$ stability than usual senses. If we impose some simple conditions for these functions, we can get the usual boundedness, exponential stability and $H_{\infty}$ stability of the solution. For example, if conditions

$$
c|x|^{p} \leq W_{0}(x, t), \quad \bar{c}|x|^{q} \leq W_{1}(x, t),
$$

hold for some positive constants $c, p, \bar{c}, q$, then we have

$$
\begin{gathered}
\limsup _{t \rightarrow \infty} E\left|\boldsymbol{x}\left(t, \boldsymbol{\zeta}, i_{0}\right)\right|^{p} \leq \frac{\alpha_{0}}{c \varepsilon_{0}}, \\
\limsup _{t \rightarrow \infty} \frac{1}{t} \int_{0}^{t} E\left|\boldsymbol{x}\left(s, \boldsymbol{\zeta}, i_{0}\right)\right|^{q} \mathrm{~d} s \leq \frac{\alpha_{0}}{\bar{c}\left(\alpha_{1}-\beta_{1}\right)},
\end{gathered}
$$

Further, let $\alpha_{0}=0$ and (3.27) hold, we obtain

$$
\begin{gathered}
\limsup _{t \rightarrow \infty} \frac{1}{t} \log \left(E\left|\boldsymbol{x}\left(t, \boldsymbol{\zeta}, i_{0}\right)\right|^{p}\right) \leq-\varepsilon_{0}, \\
\int_{0}^{\infty} E\left|\boldsymbol{x}\left(s, \boldsymbol{\zeta}, i_{0}\right)\right|^{q} \mathrm{~d} s<\infty
\end{gathered}
$$




$$
\begin{gathered}
\limsup _{t \rightarrow \infty} \frac{1}{t} \log \left(\left|\boldsymbol{x}\left(t, \boldsymbol{\zeta}, i_{0}\right)\right|\right) \leq-\frac{\varepsilon_{0}}{p}, \quad \text { a.s. } \\
\int_{0}^{\infty}\left|\boldsymbol{x}\left(s, \boldsymbol{\zeta}, i_{0}\right)\right|^{q} \mathrm{~d} s<\infty . \quad \text { a.s. }
\end{gathered}
$$

Obviously, assertion (3.28) is the usual pth moment asymptotic boundedness of the solution (see [13], [14]), assertions (3.30) and (3.32) are the usual pth moment exponential stability and almost sure exponential stability of the solution respectively (see [13], [14]), and assertions (3.31) and (3.33) show the usual $H_{\infty}$ stability of the solution (see [19]).

2) From Remark 1, the results 1) and 2) of Theorem 3.1 imply that of Theorem 3.1 in [3], and the analysis methods are similar. The main differences are that we have to deal with the terms of $\int_{-\tau}^{0} W_{j}(\varphi(\theta), t+\theta) d \eta_{j}(\theta)(j=1,2, \cdots, L)$ in our case. We mainly use integral inequalities (3.13) and (3.17). We also prove the almost sure asymptotic stability of the solution, which did not be studied for SDDEs case in [3].

3) When $W_{0}(x, t)=c|x|^{p}, W_{1}(x, t)=\bar{c}|x|^{p}, \alpha_{j}=\beta_{j}=0, j=2,3, \cdots, L$, the result 1) of Theorem 3.1 in this paper becomes that of Theorem 8.4 on asymptotic boundedness in [13].

$\mathrm{Hu}$, Mao and Shen [3] introduce two auxiliary functions $U_{1}, U_{2}$ to dominate different Lyapunov functions for different modes, and assume that the diffusion operators in all modes are controlled by function $U_{2}$. When considering different Lyapunov functions for different modes of SFDEs with Markovian switching, we may design two auxiliary functions $W_{0}, W_{1}$ to dominate all Lyapunov functions, and we relax one function $W_{1}$ to the multiple auxiliary functions $W_{j}(j=1,2, \cdots, L)$ to dominate the diffusion operators in all modes.

Next, we give an example to illustrate our theorem.

Example 3.1. Let us consider a scalar SFDE with Markovian switching

$$
\mathrm{d} \boldsymbol{x}(t)=\boldsymbol{f}\left(\boldsymbol{x}_{t}, t, r(t)\right) \mathrm{d} t+\mathbf{g}\left(\boldsymbol{x}_{t}, t, r(t)\right) \mathrm{d} B(t),
$$

where $B(t)$ is a scalar Brownian motion, $r(t)$ is a right-continuous Markovian chain on the state space $S=\{1,2\}$ with generator

$$
\Gamma=\left(\begin{array}{cc}
-1 & 1 \\
6 & -6
\end{array}\right),
$$

and

$$
\begin{aligned}
& \boldsymbol{f}\left(\boldsymbol{x}_{t}, t, 1\right)=-4\left(\boldsymbol{x}(t)+\boldsymbol{x}^{3}(t)+\boldsymbol{x}^{5}(t)\right), \quad \boldsymbol{f}\left(\boldsymbol{x}_{t}, t, 2\right)=\frac{1}{4} \boldsymbol{x}(t)-\boldsymbol{x}^{3}(t), \\
& \mathbf{g}\left(\boldsymbol{x}_{t}, t, 1\right)=\int_{-1}^{0}|\boldsymbol{x}(t+\theta)|^{3} \mathrm{~d} \eta(\theta)+\int_{-1}^{0}|\boldsymbol{x}(t+\theta)|^{2} \mathrm{~d} \eta(\theta), \quad \mathbf{g}\left(\boldsymbol{x}_{t}, t, 2\right)=\frac{1}{2} \int_{-1}^{0}|\boldsymbol{x}(t+\theta)| \mathrm{d} \eta(\theta),
\end{aligned}
$$

where $\eta$ is a probability measure on $[-1,0]$, which satisfies $\int_{-1}^{0} d \eta(\theta)=1$. 
As to system (3.34), take different Lyapunov functions for the two modes

$$
V(x, t, i)=\left\{\begin{array}{ll}
x^{2}, & i=1 \\
2\left(x^{2}+x^{6}\right), & i=2
\end{array},\right.
$$

for all $(x, t) \in R \times[-\tau,+\infty)$, and we compute that

$$
\begin{aligned}
& \mathscr{L} V\left(\boldsymbol{x}_{t}, t, 1\right)=2 x^{T} \boldsymbol{f}\left(\boldsymbol{x}_{t}, t, 1\right)+\mathbf{g}^{2}\left(\boldsymbol{x}_{t}, t, 1\right)+\sum_{j=1}^{2} \gamma_{1 j} V(\boldsymbol{x}(t), t, j) \\
& \leq-6\left(x^{2}+x^{6}\right)+2 \int_{-1}^{0}\left(|\boldsymbol{x}(t+\theta)|^{2}+|\boldsymbol{x}(t+\theta)|^{6}\right) \mathrm{d} \eta(\theta)-8 x^{4}+2 \int_{-1}^{0}|\boldsymbol{x}(t+\theta)|^{4} \mathrm{~d} \eta(\theta), \\
& \mathscr{L} V\left(\boldsymbol{x}_{t}, t, 2\right)=2\left(2 x+6 x^{5}\right)^{T} \boldsymbol{f}\left(\boldsymbol{x}_{t}, t, 2\right)+\frac{1}{2} \mathbf{g}^{T}\left(\boldsymbol{x}_{t}, t, 2\right)\left(4+60 x^{4}\right) \mathbf{g}\left(\boldsymbol{x}_{t}, t, 2\right)+\sum_{j=1}^{2} \gamma_{2 j} V(\boldsymbol{x}(t), t, j) \\
& \leq-4\left(x^{2}+x^{6}\right)+\frac{5}{2} \int_{-1}^{0}\left(|\boldsymbol{x}(t+\theta)|^{2}+|\boldsymbol{x}(t+\theta)|^{6}\right) \mathrm{d} \eta(\theta)-4 x^{4}-12 x^{8} .
\end{aligned}
$$

That is, for $i=1,2$,

$$
\mathscr{L} V\left(\boldsymbol{x}_{t}, t, i\right) \leq-4\left(x^{2}+x^{6}\right)+\frac{5}{2} \int_{-1}^{0}\left(|\boldsymbol{x}(t+\theta)|^{2}+|\boldsymbol{x}(t+\theta)|^{6}\right) \mathrm{d} \eta(\theta)-4 x^{4}+2 \int_{-1}^{0}|\boldsymbol{x}(t+\theta)|^{4} \mathrm{~d} \eta(\theta) .
$$

Define $W_{0}(x, t)=x^{2}, W_{1}(x, t)=2\left(x^{2}+x^{6}\right)$ and $W_{2}(x, t)=x^{4}$, then

$$
\begin{aligned}
& W_{0}(x, t) \leq V(x, t, i) \leq W_{1}(x, t), \\
& \mathscr{L} V\left(\boldsymbol{x}_{t}, t, i\right) \leq-2 W_{1}(x, t)+\frac{5}{4} \int_{-1}^{0} W_{1}(\boldsymbol{x}(t+\theta), t+\theta) \mathrm{d} \eta(\theta)-4 W_{2}(x, t) \\
& \quad+2 \int_{-1}^{0} W_{2}(\boldsymbol{x}(t+\theta), t+\theta) \mathrm{d} \eta(\theta),
\end{aligned}
$$

for all $x, x_{t} \in R, t \geq-1$ and $i \in S$. So we get the parameters in Assumption 3.2 as follows:

$$
\alpha_{0}=0, \alpha_{1}=2, \beta_{1}=\frac{5}{4}, \alpha_{2}=4, \beta_{2}=2 .
$$

And we compute that $\varepsilon_{0}=\sup \left\{\varepsilon>0: \varepsilon+\frac{5}{4} e^{\varepsilon}-2 \leq 0, \varepsilon \leq \log 2\right\}=0.3047$. Hence, set positive definite function $Q(\boldsymbol{x})=\boldsymbol{x}^{2} \leq W_{1}(\boldsymbol{x}, t)+W_{2}(\boldsymbol{x}, t)$, by Theorem 3.1 we get that there is a unique global solution $\boldsymbol{x}(t)$ of system (3.34), and that the global solution $\boldsymbol{x}(t)$ has the following moment properties

$$
\begin{gathered}
\limsup _{t \rightarrow \infty} \frac{\log \left(E \boldsymbol{x}^{2}(t)\right)}{t} \leq-0.3047 \\
\int_{0}^{\infty} E\left(\boldsymbol{x}^{2}(t)\right) \mathrm{d} t<\infty, \quad \int_{0}^{\infty} E\left(\boldsymbol{x}^{4}(t)\right) \mathrm{d} t<\infty, \quad \int_{0}^{\infty} E\left(\boldsymbol{x}^{6}(t)\right) \mathrm{d} t<\infty,
\end{gathered}
$$


and the following sample properties

$$
\begin{gathered}
\limsup _{t \rightarrow \infty} \frac{\log (|\boldsymbol{x}(t)|)}{t} \leq-0.1524, \quad \text { a.s. } \\
\int_{0}^{\infty} \boldsymbol{x}^{2}(t) \mathrm{d} t<\infty, \quad \int_{0}^{\infty} \boldsymbol{x}^{4}(t) \mathrm{d} t<\infty, \quad \int_{0}^{\infty} \boldsymbol{x}^{6}(t) \mathrm{d} t<\infty, \quad \text { a.s. } \\
\lim _{t \rightarrow \infty} \boldsymbol{x}(t)=0 . \quad \text { a.s. }
\end{gathered}
$$

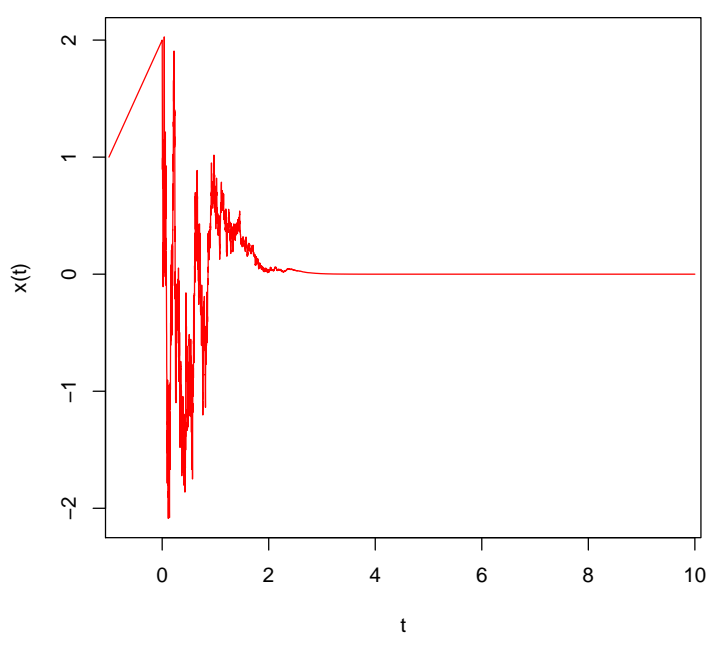

Figure 1: Simulation of the trajectory of the solution $\boldsymbol{x}(t)$ of system $(3.34)$ on $[0,10]$.

For system (3.34), take probability measure $\eta(\theta)=\frac{e^{\theta}}{1-e^{-1}}$ and initial data $\boldsymbol{\zeta}=\{\theta+2:-1 \leq$ $\theta \leq 0\}, i_{0}=1$. Based on the Euler-Maruyama scheme with step size $10^{-3}$, we give a sequence of computer simulations for system (3.34) as follows. Figure 1 illustrates the assertion (3.41) by the simulation of the trajectory of the solution $\boldsymbol{x}(t)$ of system (3.34). Figure 2 illustrates the assertions (3.37) and (3.38) by the simulations of $\frac{\log \left(E \boldsymbol{x}^{2}(t)\right)}{t}, \int_{0}^{t} E\left(\boldsymbol{x}^{2}(t)\right) \mathrm{d} t, \int_{0}^{t} E\left(\boldsymbol{x}^{4}(t)\right) \mathrm{d} t$ and $\int_{0}^{t} E\left(\boldsymbol{x}^{6}(t)\right) \mathrm{d} t$ for the solution $\boldsymbol{x}(t)$ of system (3.34). Figure 3 illustrates the assertions (3.39) and (3.40) by the simulations of the trajectories of $\frac{\log (|\boldsymbol{x}(t)|)}{t}, \int_{0}^{t} \boldsymbol{x}^{2}(t) \mathrm{d} t, \int_{0}^{t} \boldsymbol{x}^{4}(t) \mathrm{d} t$ and $\int_{0}^{t} \boldsymbol{x}^{6}(t) \mathrm{d} t$ for the solution $\boldsymbol{x}(t)$ of system (3.34).

\section{$4 \quad$ Further Results}

Assumption 3.2 in Section 3 requires that $\alpha_{0}, \alpha_{j}, \beta_{j}(j=1,2, \cdots, L)$ be constants. However, many SFDEs with Markovian switching do not satisfy these conditions. Let us see an example

$$
\mathrm{d} \boldsymbol{x}(t)=\boldsymbol{f}\left(\boldsymbol{x}_{t}, t, r(t)\right) \mathrm{d} t+\mathbf{g}\left(\boldsymbol{x}_{t}, t, r(t)\right) \mathrm{d} B(t),
$$



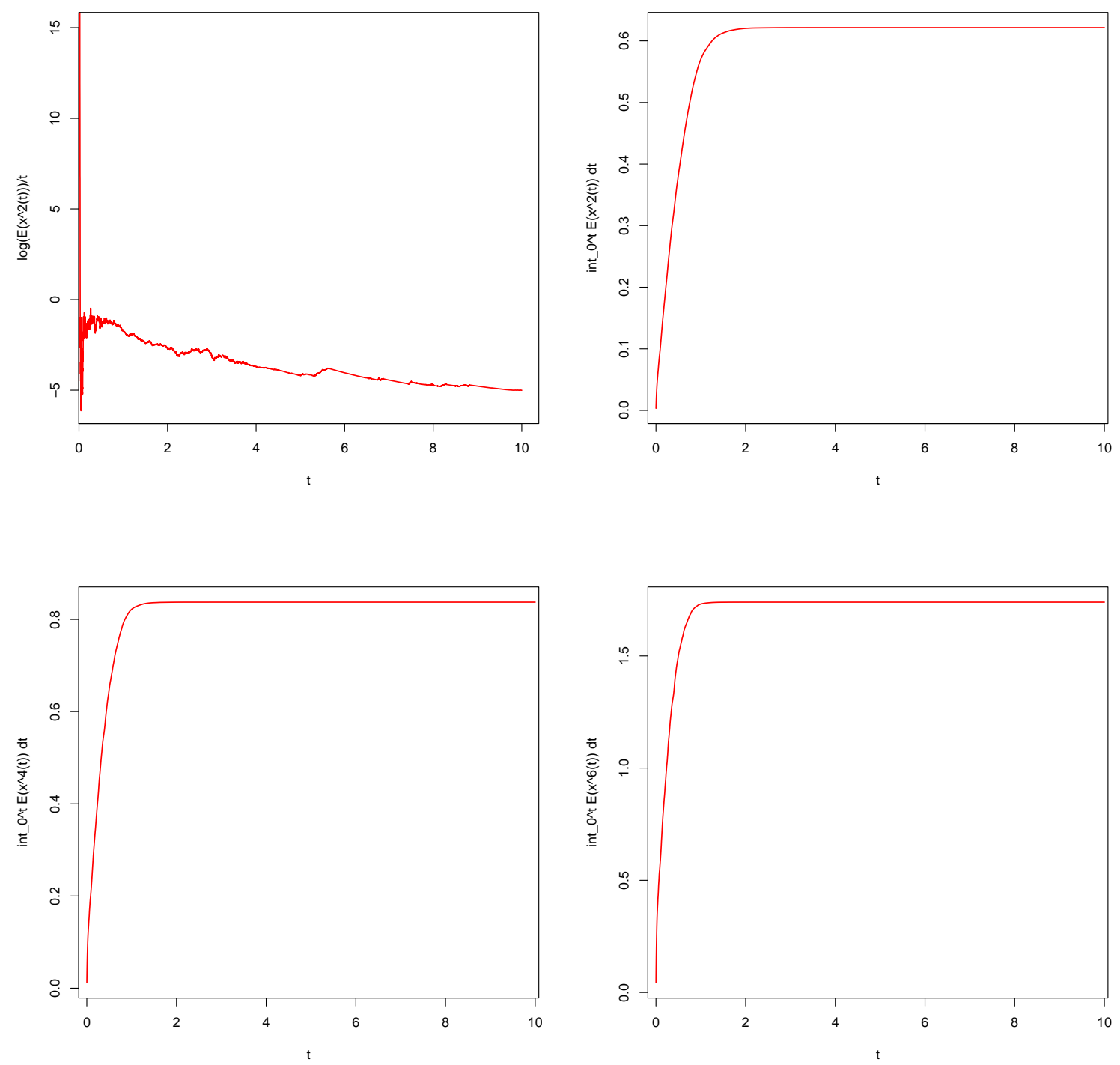

Figure 2: Simulations of $\log \left(E \boldsymbol{x}^{2}(t)\right) / t, \int_{0}^{t} E\left(\boldsymbol{x}^{2}(t)\right) \mathrm{d} t, \int_{0}^{t} E\left(\boldsymbol{x}^{4}(t)\right) \mathrm{d} t$ and $\int_{0}^{t} E\left(\boldsymbol{x}^{6}(t)\right) \mathrm{d} t$ for the solution $\boldsymbol{x}(t)$ of system (3.34) on [0, 10] and sample size 200. 

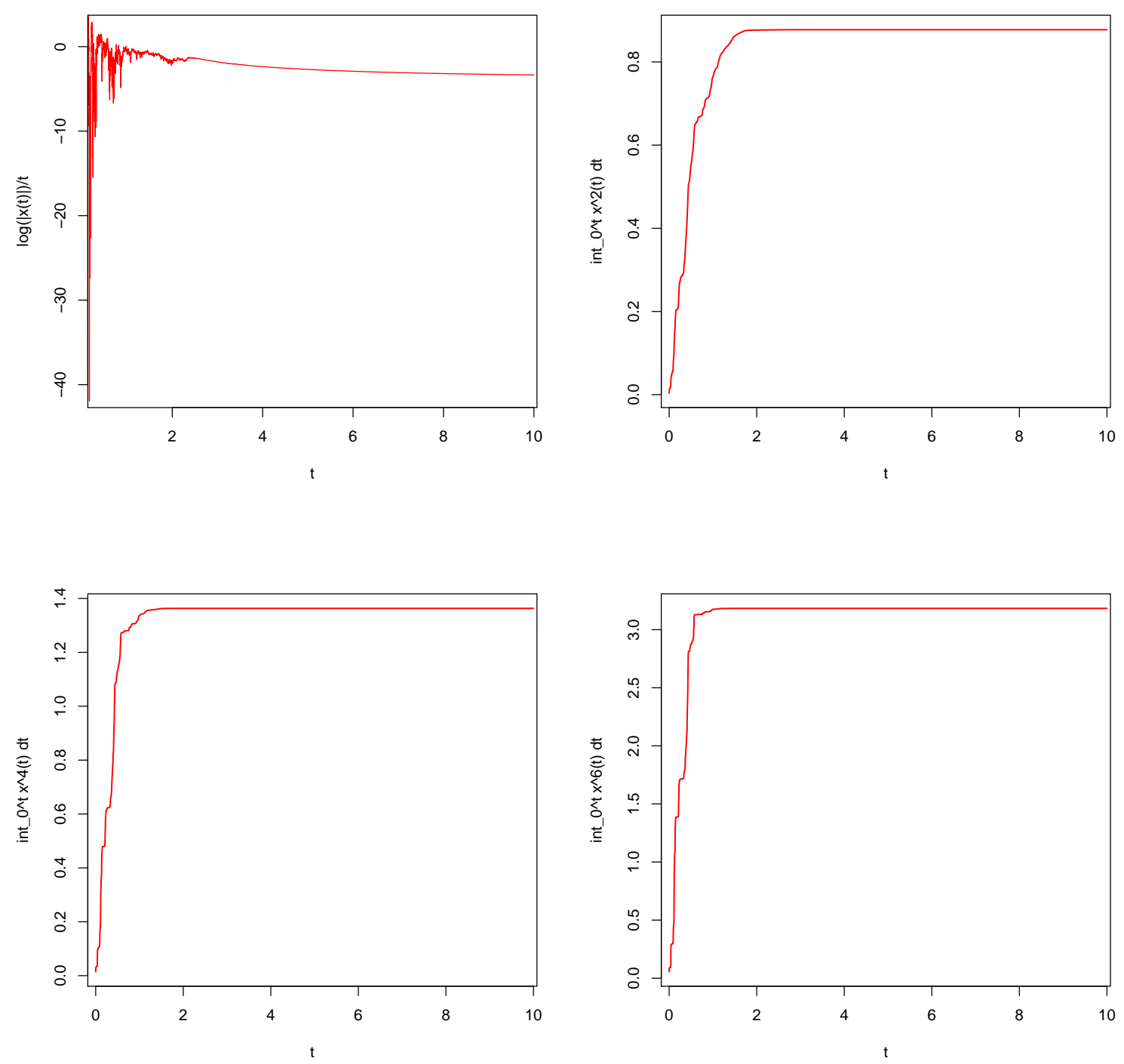

Figure 3: Simulations of the trajectories of $\log (|\boldsymbol{x}(t)|) / t, \int_{0}^{t} \boldsymbol{x}^{2}(t) \mathrm{d} t, \int_{0}^{t} \boldsymbol{x}^{4}(t) \mathrm{d} t$ and $\int_{0}^{t} \boldsymbol{x}^{6}(t) \mathrm{d} t$ for the solution $\boldsymbol{x}(t)$ of system (3.34) on [0, 10]. 
where $B(t), r(t)$ are the same as defined in system (3.34), and the coefficients are time-varying as follows:

$\boldsymbol{f}\left(\boldsymbol{x}_{t}, t, 1\right)=-4\left(1+\frac{1}{1+t}\right)\left(\boldsymbol{x}(t)+\boldsymbol{x}^{3}(t)+\boldsymbol{x}^{5}(t)\right), \quad \boldsymbol{f}\left(\boldsymbol{x}_{t}, t, 2\right)=\left(\frac{1}{4}-\frac{1}{1+t}\right) \boldsymbol{x}(t)-\frac{1}{1+t} \boldsymbol{x}^{3}(t)$,

$\mathbf{g}\left(\boldsymbol{x}_{t}, t, 1\right)=\sqrt{\frac{1}{1+t}}\left(2 e^{-t} \int_{-\tau}^{0}|\boldsymbol{x}(t+\theta)|^{\frac{1}{2}} \mathrm{~d} \eta(\theta)+\int_{-\tau}^{0}|\boldsymbol{x}(t+\theta)|^{3} \mathrm{~d} \eta(\theta)\right)$,

$\mathbf{g}\left(\boldsymbol{x}_{t}, t, 2\right)=\frac{1}{2} \sqrt{\frac{1}{1+t}} \int_{-\tau}^{0}|\boldsymbol{x}(t+\theta)| \mathrm{d} \eta(\theta)$,

where $\eta$ is a probability measure on $[-\tau, 0]$, which satisfies $\int_{-\tau}^{0} d \eta(\theta)=1$.

We easily see that all existing results and Theorem 3.1 in Section 3 can not be directly used to system (4.1) for the existence of time-varying coefficients.

As to system (4.1), using the same definition of $V(x, t, i)$ as (3.36), we compute that

$$
\begin{aligned}
& \mathscr{L} V\left(\boldsymbol{x}_{t}, t, 1\right)=2 x^{T} \boldsymbol{f}\left(\boldsymbol{x}_{t}, t, 1\right)+\mathbf{g}^{2}\left(\boldsymbol{x}_{t}, t, 1\right)+\sum_{j=1}^{2} \gamma_{1 j} V(\boldsymbol{x}(t), t, j) \\
& \leq-8 \frac{1}{1+t}\left(x^{2}+x^{6}\right)+3 \frac{1}{1+t} \int_{-\tau}^{0}\left(|\boldsymbol{x}(t+\theta)|^{2}+|\boldsymbol{x}(t+\theta)|^{6}\right) \mathrm{d} \eta(\theta)-8 \frac{1}{1+t} x^{4}+3 e^{-4 t}, \\
& \mathscr{L} V\left(\boldsymbol{x}_{t}, t, 2\right)=2\left(2 x+6 x^{5}\right)^{T} \boldsymbol{f}\left(\boldsymbol{x}_{t}, t, 2\right)+\frac{1}{2} \mathbf{g}^{T}\left(\boldsymbol{x}_{t}, t, 2\right)\left(4+60 x^{4}\right) \mathbf{g}\left(\boldsymbol{x}_{t}, t, 2\right)+\sum_{j=1}^{2} \gamma_{2 j} V(\boldsymbol{x}(t), t, j) \\
& \leq-4 \frac{1}{1+t}\left(x^{2}+x^{6}\right)+\frac{5}{2} \frac{1}{1+t} \int_{-\tau}^{0}\left(|\boldsymbol{x}(t+\theta)|^{2}+|\boldsymbol{x}(t+\theta)|^{6}\right) \mathrm{d} \eta(\theta)-4 \frac{1}{1+t} x^{4}-12 \frac{1}{1+t} x^{8} .
\end{aligned}
$$

That is, for $i=1,2$,

$\mathscr{L} V\left(\boldsymbol{x}_{t}, t, i\right) \leq-4 \frac{1}{1+t}\left(x^{2}+x^{6}\right)+3 \frac{1}{1+t} \int_{-\tau}^{0}\left(|\boldsymbol{x}(t+\theta)|^{2}+|\boldsymbol{x}(t+\theta)|^{6}\right) \mathrm{d} \eta(\theta)-4 \frac{1}{1+t} x^{4}+3 e^{-4 t}$.

Hence, define $W_{0}(x, t)=x^{2}, W_{1}(x, t)=2\left(x^{2}+x^{6}\right)$ and $W_{2}(x, t)=x^{4}$, then

$$
\begin{aligned}
W_{0}(x, t) \leq V(x, t, i) \leq & W_{1}(x, t), \\
\mathscr{L} V\left(\boldsymbol{x}_{t}, t, i\right) \leq & 3 e^{-4 t}-2 \frac{1}{1+t} W_{1}(x, t)+\frac{3}{2} \frac{1}{1+t} \int_{-\tau}^{0} W_{1}(\boldsymbol{x}(t+\theta), t+\theta) \mathrm{d} \eta(\theta) \\
& \quad 4 \frac{1}{1+t} W_{2}(x, t)+\frac{1}{1+t} \int_{-\tau}^{0} W_{2}(\boldsymbol{x}(t+\theta), t+\theta) \mathrm{d} \eta(\theta),
\end{aligned}
$$

for all $x, x_{t} \in R, t \geq-\tau$ and $i \in S$.

Then it is natural to ask the following questions: Is there a unique global solution if a SFDE with Markovian switching obeys some conditions similar to (4.2)? If yes, whether the SFDE with Markovian switching has the properties of the asymptotic stability and boundedness? So, another aim of this paper is to answer these questions above. To do these, we extend the idea of the diffusion operators dominated by multiple auxiliary functions with constant coefficients in Theorem 3.1 to that in the case of time-varying coefficients.

Motivated by system (4.1) discussed above, we propose another new assumption. 
Assumption 4.1. Let $b_{0}(t), b_{j}(t)(j=1,2, \cdots, L)$ be continuous functions from $[-\tau, \infty)$ to $R_{+}$satisfying the following conditions: $\int_{0}^{\infty} b_{0}(t) \mathrm{d} t<\infty, \int_{0}^{\infty} b_{1}(t) \mathrm{d} t=\infty$, functions $b_{j}(t)(j=$ $1,2, \cdots, L)$ are monotonically non-increasing and bounded by 1 . And let all conditions in Assumption 3.2 hold except (3.3) is replaced by

$$
\mathscr{L} V(\boldsymbol{\varphi}, t, i) \leq b_{0}(t)+\sum_{j=1}^{L}\left[-\alpha_{j} b_{j}(t) W_{j}(\boldsymbol{\varphi}(0), t)+\beta_{j} b_{j}(t) \int_{-\tau}^{0} W_{j}(\boldsymbol{\varphi}(\theta), t+\theta) d \eta_{j}(\theta)\right],
$$

for all $i \in S$ and $\varphi \in C\left([-\tau, 0] ; R^{n}\right), t \in R_{+}$.

Remark 3. Note Assumption 4.1 is different from Assumption 3.2. The difference looks "small", but it's significant in the two following aspects:

1) Because of the existence of $b_{j}(t)(j=0,1, \cdots, L)$, Assumption 4.1 is more adapt to non-autonomous systems;

2) The requirement of $\alpha_{0}$ in Assumption 3.2 is different from that of $b_{0}(t)$ in Assumption 4.1. Of course, Assumption 4.1 with $b_{0}(t)=0$ and $b_{j}(t)=1(j=1,2, \cdots, L)$, reduces to the case of Assumption 3.2 with $\alpha_{0}=0$, namely, Assumption 3.2 with $\alpha_{0}=0$ is only a special case of Assumption 4.1.

Since the coefficients of $W_{j}(j=1, \cdots, L)$ in (4.3) are time-varying, it is said to be the case of multiple auxiliary functions with time-varying coefficients for convenience. Next, we state our main results of SFDEs with Markovian switching in this case.

Theorem 4.1. Under Assumptions 3.1 and 4.1, we have the following assertions:

1) For any given initial data $\boldsymbol{\zeta} \in C$ and $i_{0} \in S$, there is a unique global solution $\boldsymbol{x}\left(t, \boldsymbol{\zeta}, i_{0}\right)$ of system (2.1) on $t \geq-\tau$, which satisfies the following moment properties

$$
\begin{gathered}
\sup _{-\tau \leq t<\infty} E W_{0}\left(\boldsymbol{x}\left(t, \boldsymbol{\zeta}, i_{0}\right), t\right)<\infty, \\
\int_{0}^{\infty} b_{j}(s) E W_{j}\left(\boldsymbol{x}\left(s, \boldsymbol{\zeta}, i_{0}\right), s\right) \mathrm{d} s<\infty, \quad j=1,2, \cdots, L \\
E W_{0}\left(\boldsymbol{x}\left(t, \boldsymbol{\zeta}, i_{0}\right), t\right) \leq c_{2} e^{-\varepsilon_{0} \int_{0}^{t} b_{1}(h) \mathrm{d} h}+\int_{0}^{t} e^{-\varepsilon_{0} \int_{s}^{t} b_{1}(h) \mathrm{d} h} b_{0}(s) \mathrm{d} s \\
\lim _{t \rightarrow \infty} E W_{0}\left(\boldsymbol{x}\left(t, \boldsymbol{\zeta}, i_{0}\right), t\right)=0 .
\end{gathered}
$$

and the following sample properties

$$
\begin{gathered}
\sup _{-\tau \leq t<\infty} \boldsymbol{x}\left(t, \boldsymbol{\zeta}, i_{0}\right)<\infty, \quad \text { a.s. } \\
\int_{0}^{\infty} b_{j}(s) W_{j}\left(\boldsymbol{x}\left(s, \boldsymbol{\zeta}, i_{0}\right), s\right) \mathrm{d} s<\infty, \quad \text { a.s. } \quad j=1,2, \cdots, L
\end{gathered}
$$


where $c_{2}$ is a finite positive constant, $\varepsilon_{0}$ is the same as defined in Theorem 3.1.

2) Moreover, if $b_{0}(t)=0$, the global solution $\boldsymbol{x}\left(t, \boldsymbol{\zeta}, i_{0}\right)$ has the following moment property

$$
\limsup _{t \rightarrow \infty} \frac{1}{t} \log \left(E W_{0}\left(\boldsymbol{x}\left(t, \boldsymbol{\zeta}, i_{0}\right), t\right)\right) \leq-\varepsilon_{0} \liminf _{t \rightarrow \infty} \frac{\int_{0}^{t} b_{1}(h) \mathrm{d} h}{t},
$$

and the following sample property

$$
\limsup _{t \rightarrow \infty} \frac{1}{t} \log \left(W_{0}\left(\boldsymbol{x}\left(t, \boldsymbol{\zeta}, i_{0}\right), t\right)\right) \leq-\varepsilon_{0} \liminf _{t \rightarrow \infty} \frac{\int_{0}^{t} b_{1}(h) \mathrm{d} h}{t} . \quad \text { a.s. }
$$

3) If furthermore there exists a continuous positive definite function $G \in C\left(R^{n} ; R_{+}\right)$and a continuous function $\psi(\cdot) \in \Psi\left(R_{+} ; R_{+}\right)$such that $\psi(t) G(\boldsymbol{x}) \leq \sum_{j=1}^{L} b_{j}(t) W_{j}(\boldsymbol{x}, t)$ for all $(\boldsymbol{x}, t) \in R^{n} \times[-\tau, \infty)$, then

$$
\lim _{t \rightarrow \infty} \boldsymbol{x}\left(t, \boldsymbol{\zeta}, i_{0}\right)=0 . \quad \text { a.s. }
$$

Proof. 1) The proof of the existence and uniqueness of the global solution $\boldsymbol{x}\left(t, \boldsymbol{\zeta}, i_{0}\right)$ of system (2.1) is essentially similar to that of Theorem 3.1, so we omit it. For the sake of simplicity, write $\boldsymbol{x}(t)=\boldsymbol{x}\left(t, \boldsymbol{\zeta}, i_{0}\right)$. Using the generalized Itô's formula to $e^{\varepsilon \int_{0}^{t} b_{1}(h) \mathrm{d} h} V(\boldsymbol{x}(t), t, r(t)), \varepsilon \in$ $\left[0, \varepsilon_{0}\right]$, and from condition (3.2), we get that, for any $t \geq 0$,

$$
\begin{aligned}
& e^{\varepsilon \int_{0}^{t} b_{1}(h) \mathrm{d} h} V(\boldsymbol{x}(t), t, r(t)) \\
& =V(\boldsymbol{x}(0), 0, r(0))+\int_{0}^{t} e^{\varepsilon \int_{0}^{s} b_{1}(h) \mathrm{d} h}\left(\varepsilon b_{1}(s) V(\boldsymbol{x}(s), s, r(s))+\mathscr{L} V\left(\boldsymbol{x}_{s}, s, r(s)\right)\right) \mathrm{d} s+M_{1}(t) \\
& \leq W_{1}(\boldsymbol{x}(0), 0)+\int_{0}^{t} e^{\varepsilon \int_{0}^{s} b_{1}(h) \mathrm{d} h}\left[b_{0}(s)-\left(\alpha_{1}-\varepsilon\right) b_{1}(s) W_{1}(\boldsymbol{x}(s), s)\right. \\
& +\beta_{1} b_{1}(s) \int_{-\tau}^{0} W_{1}(\boldsymbol{x}(s+\theta), s+\theta) d \eta_{1}(\theta)+\sum_{j=2}^{L}\left[-\alpha_{j} b_{j}(s) W_{j}(\boldsymbol{x}(s), s)\right. \\
& \left.\left.+\beta_{j} b_{j}(s) \int_{-\tau}^{0} W_{j}(\boldsymbol{x}(s+\theta), s+\theta) d \eta_{j}(\theta)\right]\right] \mathrm{d} s+M_{1}(t) \\
& \leq W_{1}(\boldsymbol{x}(0), 0)+\int_{0}^{t} e^{\varepsilon \int_{0}^{s} b_{1}(h) \mathrm{d} h}\left[b_{0}(s)-\left(\alpha_{1}-\varepsilon-\beta_{1} e^{\varepsilon \tau}\right) b_{1}(s) W_{1}(\boldsymbol{x}(s), s)+\beta_{1} b_{1}(s) J_{1}^{\prime}\right. \\
& \left.+\sum_{j=2}^{L}\left[-\left(\alpha_{j}-\beta_{j} e^{\varepsilon \tau}\right) b_{j}(s) W_{j}(\boldsymbol{x}(s), s)+\beta_{j} b_{j}(s) J_{j}^{\prime}\right]\right] \mathrm{d} s+M_{1}(t),
\end{aligned}
$$

where $M_{1}(t)$ is a local martingale with the initial value $M_{1}(0)=0, J_{j}^{\prime}=\int_{-\tau}^{0} W_{j}(\boldsymbol{x}(s+\theta), s+$ $\theta) \mathrm{d} \eta_{j}(\theta)-e^{\varepsilon \tau} W_{j}(\boldsymbol{x}(s), s), j=1,2, \cdots L$.

By virtue of the fact that

$$
\int_{0}^{t} e^{\varepsilon \int_{0}^{s} b_{1}(h) \mathrm{d} h} b_{j}(s) J_{j}^{\prime} \mathrm{d} s
$$




$$
\begin{aligned}
& \leq \int_{-\tau}^{0} \int_{-\tau}^{t} e^{\varepsilon \int_{0}^{r-\theta} b_{1}(h) \mathrm{d} h} b_{j}(r-\theta) W_{j}(\boldsymbol{x}(r), r) \mathrm{d} r \mathrm{~d} \eta_{j}(\theta)-e^{\varepsilon \tau} \int_{0}^{t} e^{\varepsilon \int_{0}^{s} b_{1}(h) \mathrm{d} h} b_{j}(s) W_{j}(\boldsymbol{x}(s), s) \mathrm{d} s \\
& \leq \int_{-\tau}^{0} \int_{-\tau}^{t} e^{\varepsilon\left(\int_{0}^{r} b_{1}(h) \mathrm{d} h+\int_{r}^{r-\theta} b_{1}(h) \mathrm{d} h\right)} b_{j}(r) W_{j}(\boldsymbol{x}(r), r) \mathrm{d} r \mathrm{~d} \eta_{j}(\theta)-e^{\varepsilon \tau} \int_{0}^{t} e^{\varepsilon \int_{0}^{s} b_{1}(h) \mathrm{d} h} b_{j}(s) W_{j}(\boldsymbol{x}(s), s) \mathrm{d} s \\
& \leq e^{\varepsilon \tau} \int_{-\tau}^{0} e^{\varepsilon \int_{0}^{s} b_{1}(h) \mathrm{d} h} b_{j}(s) W_{j}(\boldsymbol{x}(s), s) \mathrm{d} s
\end{aligned}
$$

for $j=1,2, \cdots, L,(4.13)$ can be rewritten as

$$
\begin{aligned}
& e^{\varepsilon \int_{0}^{t} b_{1}(h) \mathrm{d} h} V(\boldsymbol{x}(t), t, r(t)) \\
& \leq W_{1}(\boldsymbol{x}(0), 0)+\int_{0}^{t} e^{\varepsilon \int_{0}^{s} b_{1}(h) \mathrm{d} h} b_{0}(s) \mathrm{d} s-\left(\alpha_{1}-\varepsilon-\beta_{1} e^{\varepsilon \tau}\right) \int_{0}^{t} e^{\varepsilon \int_{0}^{s} b_{1}(h) \mathrm{d} h} b_{1}(s) W_{1}(\boldsymbol{x}(s), s) \mathrm{d} s \\
&-\sum_{j=2}^{L}\left(\alpha_{j}-\beta_{j} e^{\varepsilon \tau}\right) \int_{0}^{t} e^{\varepsilon \int_{0}^{s} b_{1}(h) \mathrm{d} h} b_{j}(s) W_{j}(\boldsymbol{x}(s), s) \mathrm{d} s \\
&+\sum_{j=1}^{L} \beta_{j} e^{\varepsilon \tau} \int_{-\tau}^{0} e^{\varepsilon \int_{0}^{s} b_{1}(h) \mathrm{d} h} b_{j}(s) W_{j}(\boldsymbol{x}(s), s) \mathrm{d} s+M_{1}(t) \\
& \leq c_{2}+\int_{0}^{t} e^{\varepsilon \int_{0}^{s} b_{1}(h) \mathrm{d} h} b_{0}(s) \mathrm{d} s-\left(\alpha_{1}-\varepsilon-\beta_{1} e^{\varepsilon \tau}\right) \int_{0}^{t} e^{\varepsilon \int_{0}^{s} b_{1}(h) \mathrm{d} h} b_{1}(s) W_{1}(\boldsymbol{x}(s), s) \mathrm{d} s \\
&-\sum_{j=2}^{L}\left(\alpha_{j}-\beta_{j} e^{\varepsilon \tau}\right) \int_{0}^{t} e^{\varepsilon \int_{0}^{s} b_{1}(h) \mathrm{d} h} b_{j}(s) W_{j}(\boldsymbol{x}(s), s) \mathrm{d} s+M_{1}(t),
\end{aligned}
$$

where $c_{2}=W_{1}(\boldsymbol{x}(0), 0)+\sum_{j=1}^{L} \beta_{j} e^{\varepsilon \tau} \int_{-\tau}^{0} e^{\varepsilon \int_{0}^{s} b_{1}(h) \mathrm{d} h} b_{j}(s) W_{j}(\boldsymbol{x}(s), s) \mathrm{d} s$. Take the expectation on both side of (4.15), and let $\varepsilon \rightarrow \varepsilon_{0}$, we get the assertion (4.6)

$$
E W_{0}(\boldsymbol{x}(t), t) \leq c_{2} e^{-\varepsilon_{0} \int_{0}^{t} b_{1}(h) \mathrm{d} h}+\int_{0}^{t} e^{-\varepsilon_{0} \int_{s}^{t} b_{1}(h) \mathrm{d} h} b_{0}(s) \mathrm{d} s,
$$

from the definition of $\varepsilon_{0}$ and condition (3.2).

From condition $\int_{0}^{\infty} b_{1}(t) \mathrm{d} t=\infty$, it is obvious that, as $t \rightarrow \infty$,

$$
e^{-\varepsilon_{0} \int_{0}^{t} b_{1}(h) \mathrm{d} h} \rightarrow 0 .
$$

On the other hand, we have that

$$
\begin{aligned}
\int_{0}^{t} e^{-\varepsilon_{0} \int_{s}^{t} b_{1}(h) \mathrm{d} h} b_{0}(s) \mathrm{d} s & =\int_{0}^{T} e^{-\varepsilon_{0} \int_{s}^{t} b_{1}(h) \mathrm{d} h} b_{0}(s) \mathrm{d} s+\int_{T}^{t} e^{-\varepsilon_{0} \int_{s}^{t} b_{1}(h) \mathrm{d} h} b_{0}(s) \mathrm{d} s \\
& \leq e^{-\varepsilon_{0} \int_{T}^{t} b_{1}(h) \mathrm{d} h} \int_{0}^{\infty} b_{0}(s) \mathrm{d} s+\int_{T}^{\infty} b_{0}(s) \mathrm{d} s
\end{aligned}
$$

For fixed $\mathrm{T}, e^{-\varepsilon_{0} \int_{T}^{t} b_{1}(h) \mathrm{d} h} \rightarrow 0$ holds, as $t \rightarrow \infty$. Then letting $T \rightarrow \infty$, we have $\int_{T}^{\infty} b_{0}(s) \mathrm{d} s \rightarrow$ 0 . So

$$
\int_{0}^{t} e^{-\varepsilon_{0} \int_{s}^{t} b_{1}(h) \mathrm{d} h} b_{0}(s) \mathrm{d} s \rightarrow 0
$$


holds, as $t \rightarrow \infty$. Therefore, we get the result (4.7)

$$
\lim _{t \rightarrow \infty} E W_{0}(\boldsymbol{x}(t), t)=0 .
$$

Letting $\varepsilon \rightarrow 0$ in (4.15), taking the expectation and then using Fubini theorem, we have

$$
E W_{0}(\boldsymbol{x}(t), t) \leq c_{2}^{\prime}+\int_{0}^{t} b_{0}(s) \mathrm{d} s-\sum_{j=1}^{L}\left(\alpha_{j}-\beta_{j}\right) \int_{0}^{t} b_{j}(s) E W_{j}(\boldsymbol{x}(s), s) \mathrm{d} s,
$$

where $c_{2}^{\prime}=E W_{1}(\boldsymbol{x}(0), 0)+\sum_{j=1}^{L} \beta_{j} E \int_{-\tau}^{0} b_{j}(s) W_{j}(\boldsymbol{x}(s), s) \mathrm{d} s$. From condition $\int_{0}^{\infty} b_{0}(t) \mathrm{d} t<$ $\infty$, we get the assertions (4.4) and (4.5)

$$
\begin{aligned}
& \sup _{-\tau \leq t<\infty} E W_{0}(\boldsymbol{x}(t), t) \leq c_{2}^{\prime}+\int_{0}^{\infty} b_{0}(s) \mathrm{d} s<\infty, \\
& \int_{0}^{\infty} b_{j}(s) E W_{j}(\boldsymbol{x}(s), s) \mathrm{d} s \leq \frac{c_{2}^{\prime}+\int_{0}^{\infty} b_{0}(s) \mathrm{d} s}{\alpha_{j}-\beta_{j}}<\infty . \quad j=1,2, \cdots, L
\end{aligned}
$$

Letting $\varepsilon \rightarrow 0,(4.15)$ becomes

$$
V(\boldsymbol{x}(t), t, r(t)) \leq c_{3}+\int_{0}^{t} b_{0}(s) \mathrm{d} s-\sum_{j=1}^{L}\left(\alpha_{j}-\beta_{j}\right) \int_{0}^{t} b_{j}(s) W_{j}(\boldsymbol{x}(s), s) \mathrm{d} s+M_{2}(t),
$$

where $c_{3}=W_{1}(\boldsymbol{x}(0), 0)+\sum_{j=1}^{L} \beta_{j} \int_{-\tau}^{0} b_{j}(s) W_{j}(\boldsymbol{x}(s), s) \mathrm{d} s, M_{2}(t)$ is a local martingale with the initial value $M_{2}(0)=0$. From Lemma 2.1, we obtain the assertions (4.9) and $\sup _{-\tau \leq t<\infty} W_{0}(\boldsymbol{x}(t), t)<$ $\infty, a . s$. From the radial unboundedness of $W_{0}(x, t)$ (see (3.1)), we directly get the assertion (4.8).

2) Next, we prove the results in the case of $b_{0}(t)=0$. (4.15) becomes

$$
e^{\varepsilon \int_{0}^{t} b_{1}(h) \mathrm{d} h} V(\boldsymbol{x}(t), t, r(t)) \leq c_{2}+M_{1}(t),
$$

where $c_{2}$ has been defined above. Taking the expectation on both side of (4.20), we have

$$
e^{\varepsilon \int_{0}^{t} b_{1}(h) \mathrm{d} h} E W_{0}(\boldsymbol{x}(t), t, r(t)) \leq c_{2} .
$$

So as $\varepsilon \rightarrow \varepsilon_{0}$, we get the result (4.10)

$$
\limsup _{t \rightarrow \infty} \frac{\log \left(E W_{0}(\boldsymbol{x}(t), t)\right)}{t} \leq-\varepsilon_{0} \liminf _{t \rightarrow \infty} \frac{\int_{0}^{t} b_{1}(h) \mathrm{d} h}{t} .
$$

Applying Lemma 2.1 to (4.20), we obtain that

$$
\limsup _{t \rightarrow \infty} e^{\varepsilon \int_{0}^{t} b_{1}(h) \mathrm{d} h} W_{0}(\boldsymbol{x}(t), t)<\infty . \quad \text { a.s. }
$$

Hence, there exists a finite positive random variable $\bar{\zeta}$ such that

$$
\sup _{0 \leq t<\infty} e^{\varepsilon \int_{0}^{t} b_{1}(h) \mathrm{d} h} W_{0}(\boldsymbol{x}(t), t)<\bar{\zeta} . \quad \text { a.s. }
$$


So as $\varepsilon \rightarrow \varepsilon_{0}$, we get the result (4.11)

$$
\limsup _{t \rightarrow \infty} \frac{\log \left(W_{0}(\boldsymbol{x}(t), t)\right)}{t} \leq-\varepsilon_{0} \liminf _{t \rightarrow \infty} \frac{\int_{0}^{t} b_{1}(h) \mathrm{d} h}{t} . \quad \text { a.s. }
$$

3) Finally, we prove the result (4.12). Similar to the proof of (3.11), the assertion (4.12) is equivalent to the assertion

$$
\limsup _{t \rightarrow \infty} G(\boldsymbol{x}(t))=0 . \quad \text { a.s. }
$$

If this is false, then there exists a constant $\delta_{1}$ such that $P(B)>3 \delta_{1}$, where $B=\{\omega$ : $\left.\limsup _{t \rightarrow \infty} G(\boldsymbol{x}(t))>2 \delta_{1}\right\}$. From the assertion (4.8), we can see that there exists a sufficiently large positive constant $h=h\left(\delta_{1}\right)$ such that $P(D)>1-\delta_{1}$, where $D=\left\{\omega: \sup _{-\tau \leq t<\infty}|\boldsymbol{x}(t)|<\right.$ $h\}$. So we easily obtain that $P(B \cap D)>2 \delta_{1}$.

For any fixed $\bar{\theta}>0$, define a sequence of stopping times by

$$
\begin{aligned}
& \sigma_{1}=\inf \left\{t \geq 0: G(\boldsymbol{x}(t)) \geq 2 \delta_{1}\right\}, \quad \sigma_{2}=\inf \left\{t \geq \sigma_{1}: G(\boldsymbol{x}(t)) \leq \delta_{1}\right\}, \\
& \sigma_{2 l+1}=\inf \left\{t \geq \sigma_{2 l}+\bar{\theta}: G(\boldsymbol{x}(t)) \geq 2 \delta_{1}\right\}, \quad l=1,2, \ldots \\
& \sigma_{2 l+2}=\inf \left\{t \geq \sigma_{2 l+1}: G(\boldsymbol{x}(t)) \leq \delta_{1}\right\} . \quad l=1,2, \ldots
\end{aligned}
$$

Similarly, we obtain $B \cap D \subset\left\{\sigma_{l}<\infty, l=1,2, \ldots\right\}$ and that, for any $T_{2}>0$,

$$
E\left(I_{B \cap D} \sup _{0 \leq t \leq T_{2}}\left|\boldsymbol{x}\left(\sigma_{2 l-1}+t\right)-\boldsymbol{x}\left(\sigma_{2 l-1}\right)\right|^{2}\right) \leq 2 T_{2} H_{h}^{2}\left(T_{2}+4\right)
$$

and that there exists a sufficiently small $\delta_{2}=\delta_{2}\left(\delta_{1}\right)>0$ such that $|G(\boldsymbol{x})-G(\boldsymbol{y})|<\delta_{1}$, when $|\boldsymbol{x}| \vee|\boldsymbol{y}| \leq h$ and $|\boldsymbol{x}-\boldsymbol{y}| \leq \delta_{2}$.

Noting that $\left\{\sup _{0 \leq t \leq T_{2}}\left|\boldsymbol{x}\left(\sigma_{2 l-1}+t\right)-\boldsymbol{x}\left(\sigma_{2 l-1}\right)\right| \leq \delta_{2}\right\} \subset\left\{\sup _{0 \leq t \leq T_{2}}\left|G\left(\boldsymbol{x}\left(\sigma_{2 l-1}+t\right)\right)-G\left(\boldsymbol{x}\left(\sigma_{2 l-1}\right)\right)\right|<\right.$ $\left.\delta_{1}\right\} \subset\left\{\sigma_{2 l}-\sigma_{2 l-1} \geq T_{2}\right\}$, we similarly choose a sufficiently small $T_{2}>0$ such that $P\left(B \cap D \cap\left\{\sup _{0 \leq t \leq T_{2}}\left|\boldsymbol{x}\left(\sigma_{2 l-1}+t\right)-\boldsymbol{x}\left(\sigma_{2 l-1}\right)\right|<\delta_{2}\right\}\right)>\delta_{1}$.

Since $\psi(t) \in \Psi\left(R_{+} ; R_{+}\right)$, then for the chosen $T_{2}>0$ there exist constants $\rho=\rho\left(T_{2}\right), T_{3}=$ $T_{3}\left(\rho, T_{2}\right)$ such that $\int_{t}^{t+T_{2}} \psi(s) \mathrm{d} s \geq \rho$, when $t \geq T_{3}$. From the definition of $\sigma_{l}$, there exists a positive integer $Q_{1}=\left[T_{3} / \bar{\theta}\right]+2$ such that, for any $\omega \in B \cap D, \sigma_{2 l-1}>T_{3}$, when $l \geq Q_{1}$. Hence, for any $\omega \in B \cap D$, we have $\int_{\sigma_{2 l-1}}^{\sigma_{2 l-1}+T_{2}} \psi(s) \mathrm{d} s \geq \rho, l \geq Q_{1}$.

On the other hand, from the assertion (4.5) and the relation of $\psi(t) G(\boldsymbol{x})$ and $\sum_{j=1}^{L} b_{j}(t) W_{j}(\boldsymbol{x}, t)$, we easily get that

$$
E \int_{0}^{\infty} \psi(s) G(\boldsymbol{x}(s)) \mathrm{d} s \leq \sum_{j=1}^{L} E \int_{0}^{\infty} b_{j}(s) W_{j}\left(\boldsymbol{x}\left(s, \boldsymbol{\zeta}, i_{0}\right), s\right) \mathrm{d} s<\infty .
$$




$$
\begin{aligned}
\text { For any } \omega \in B \cap D \cap & \left\{\sup _{0 \leq t \leq T_{2}}\left|\boldsymbol{x}\left(\sigma_{2 l-1}+t\right)-\boldsymbol{x}\left(\sigma_{2 l-1}\right)\right|<\delta_{2}\right\}, \text { we have } \\
E \int_{0}^{\infty} \psi(s) G(\boldsymbol{x}(s)) \mathrm{d} s & \geq E\left(\sum_{l=1}^{\infty} I_{\left\{\sigma_{l}<\infty\right\}} \int_{\sigma_{2 l-1}}^{\sigma_{2 l}} \psi(s) G(\boldsymbol{x}(s)) \mathrm{d} s\right) \\
& \geq \delta_{1} E\left(\sum_{l=Q_{1}}^{\infty} I_{B \cap D \cap\left\{\sup _{0 \leq t \leq T_{2}}\left|\boldsymbol{x}\left(\sigma_{2 l-1}+t\right)-\boldsymbol{x}\left(\sigma_{2 l-1}\right)\right|<\delta_{2}\right\}} \int_{\sigma_{2 l-1}}^{\sigma_{2 l}} \psi(s) \mathrm{d} s\right) \\
& \geq \delta_{1} E\left(\sum_{l=Q_{1}}^{\infty} I_{B \cap D \cap\left\{\sup _{0 \leq t \leq T_{2}}\left|\boldsymbol{x}\left(\sigma_{2 l-1}+t\right)-\boldsymbol{x}\left(\sigma_{2 l-1}\right)\right|<\delta_{2}\right\}} \int_{\sigma_{2 l-1}}^{\sigma_{2 l-1}+T_{2}} \psi(s) \mathrm{d} s\right) \\
& \geq \delta_{1} \rho \sum_{l=Q_{1}}^{\infty} \delta_{1} \\
& =\infty,
\end{aligned}
$$

which implies a contradiction with (4.24). So we get the assertion (4.12).

Remark 4. The results of Theorem 4.1 look similar to that of Theorem 3.1, but they are in fact significantly different. The contribution of Theorem 4.1 is significant in at least the following three aspects:

1) When the system is non-autonomous, it is natural to resort to Theorem 4.1 rather than Theorem 3.1;

2) Since $\Psi\left(R_{+} ; R_{+}\right)$contains many functions (see [6]), the results of Theorem 4.1 can be used to much greater fields than that of Theorem 3.1 with $\alpha_{0}=0$;

3) Because of the properties of $\Psi\left(R_{+} ; R_{+}\right)$, the techniques used in Theorem 3.1 can not be used to Theorem 4.1 directly. So we introduce some new techniques to cope with the function in $\Psi\left(R_{+} ; R_{+}\right)$.

Proposition 4.1. Let Assumptions 3.1, 4.1 hold except (4.3) is replaced by

$$
\begin{aligned}
& \mathscr{L} V(\boldsymbol{\varphi}, t, i) \leq b_{0}(t)+\sum_{j=1}^{L}\left[-\alpha_{j} b_{j}(t) W_{j}(\boldsymbol{\varphi}(0), t)+\beta_{j} b_{j}^{\prime}(t) \int_{-\tau}^{0} W_{j}^{\prime}(\boldsymbol{\varphi}(\theta), t+\theta) d \eta_{j}(\theta)\right], \\
& b_{j}(t) \geq b_{j}^{\prime}(t), \quad W_{j}(\boldsymbol{\varphi}, t) \geq W_{j}^{\prime}(\boldsymbol{\varphi}, t), \quad j=1,2, \cdots, L
\end{aligned}
$$

for all $i \in S$ and $\varphi \in C\left([-\tau, 0] ; R^{n}\right), t \in R_{+}$, where $b_{j}^{\prime}(t) \in C\left([-\tau, \infty), R_{+}\right), W_{j}^{\prime} \in C\left(R^{n} \times\right.$ $\left.[-\tau, \infty) ; R_{+}\right)$. Then all results of Theorem 4.1 hold.

Finally, we give the following example to illustrate our theorem.

Example 4.1. Let us continue to use the notations of system (4.1). Further we set $\tau=0.2$. By (4.2) and $W_{0}(x, t)=x^{2}, W_{1}(x, t)=2\left(x^{2}+x^{6}\right), W_{2}(x, t)=x^{4}$, we note that $W_{0}(x, t)$ satisfies (3.1), and get the parameters in Assumption 4.1 as follows:

$$
b_{0}(t)=3 e^{-4 t}, b_{1}(t)=b_{2}(t)=\frac{1}{1+t}, \alpha_{1}=2, \beta_{1}=\frac{3}{2}, \alpha_{2}=4, \beta_{2}=1 .
$$


And we compute that $\varepsilon_{0}=\sup \left\{\varepsilon>0: \varepsilon+\frac{3}{2} e^{0.2 \varepsilon}-2 \leq 0, \varepsilon \leq 10 \log 2\right\}=0.3812$. Hence, from Theorem 4.1 there is a unique global solution $\boldsymbol{x}(t)$ of system (4.1). And we get that the global solution $\boldsymbol{x}(t)$ of system (4.1) has the following moment properties

$$
\begin{gathered}
\sup _{-0.2 \leq t<\infty} E\left(\boldsymbol{x}^{2}(t)\right)<\infty \\
\int_{0}^{\infty} \frac{1}{1+t} E\left(\boldsymbol{x}^{2}(t)\right) \mathrm{d} t<\infty, \int_{0}^{\infty} \frac{1}{1+t} E\left(\boldsymbol{x}^{4}(t)\right) \mathrm{d} t<\infty, \int_{0}^{\infty} \frac{1}{1+t} E\left(\boldsymbol{x}^{6}(t)\right) \mathrm{d} t<\infty \\
E\left(\boldsymbol{x}^{2}(t)\right) \leq c_{2} e^{-0.3812 \log (1+t)}+3 e^{-0.3812 \log (1+t)} \int_{0}^{t} e^{0.3812 \log (1+s)-2 s} \mathrm{~d} s \\
\lim _{t \rightarrow \infty} E\left(\boldsymbol{x}^{2}(t)\right)=0
\end{gathered}
$$

and the following sample properties

$$
\begin{gathered}
\sup _{-0.2 \leq t<\infty}|\boldsymbol{x}(t)|<\infty, \\
\int_{0}^{\infty} \frac{1}{1+t} x^{2}(t) \mathrm{d} t<\infty, \quad \int_{0}^{\infty} \frac{1}{1+t} x^{4}(t) \mathrm{d} t<\infty, \quad \int_{0}^{\infty} \frac{1}{1+t} x^{6}(t) \mathrm{d} t<\infty, \quad \text { a.s. }
\end{gathered}
$$

where $c_{2}$ is a finite positive constant.

Set positive definite function $G(\boldsymbol{x})=\boldsymbol{x}^{2}$ and $\psi(t)=\frac{1}{1+t} \in \Psi\left(R_{+} ; R_{+}\right)$, then $\psi(t) G(\boldsymbol{x}) \leq$ $b_{1}(t) W_{1}(\boldsymbol{x}, t)+b_{2}(t) W_{2}(\boldsymbol{x}, t)$, and from Theorem 4.1 we get that

$$
\lim _{t \rightarrow \infty} \boldsymbol{x}\left(t, \boldsymbol{\zeta}, i_{0}\right)=0 . \quad \text { a.s. }
$$

For system (4.1), take probability measure $\eta(\theta)=\frac{e^{\theta}-e^{-0.2}}{1-e^{-0.2}}$ and initial data $\boldsymbol{\zeta}=\{\theta+2$ : $-0.2 \leq \theta \leq 0\}, i_{0}=1$. Based on the Euler-Maruyama scheme with step size $10^{-3}$, we give a sequence of computer simulations for system (4.1) as follows. Figure 4 illustrates the assertions (4.25) and (4.26) by the simulations of $E\left(\boldsymbol{x}^{2}(t)\right), \int_{0}^{t} \frac{1}{1+t} E\left(\boldsymbol{x}^{2}(t)\right) \mathrm{d} t, \int_{0}^{t} \frac{1}{1+t} E\left(\boldsymbol{x}^{4}(t)\right) \mathrm{d} t$ and $\int_{0}^{t} \frac{1}{1+t} E\left(\boldsymbol{x}^{6}(t)\right) \mathrm{d} t$ for the solution $\boldsymbol{x}(t)$ of system (4.1). Figure 5 illustrates the assertions (4.27) and (4.28) by the simulations of the trajectories of the solution $\boldsymbol{x}(t)$ of system (4.1), $\int_{0}^{t} \frac{1}{1+t} \boldsymbol{x}^{2}(t) \mathrm{d} t, \int_{0}^{t} \frac{1}{1+t} \boldsymbol{x}^{4}(t) \mathrm{d} t$ and $\int_{0}^{t} \frac{1}{1+t} \boldsymbol{x}^{6}(t) \mathrm{d} t$.

\section{Conclusions}

Motivated by Hu, Mao and Shen [3], we have used the method of multiple Lyapunov functions to study the asymptotic boundedness and stability of the SFDE with Markovian switching. Different type of Lyapunov functions for different mode has been used to deal with the structure difference of the underlying SFDE in different mode. It is in this way that our results on the boundedness and stability are more general than the existing results in this area. Several examples and computer simulations have been used to illustrate our new results. 

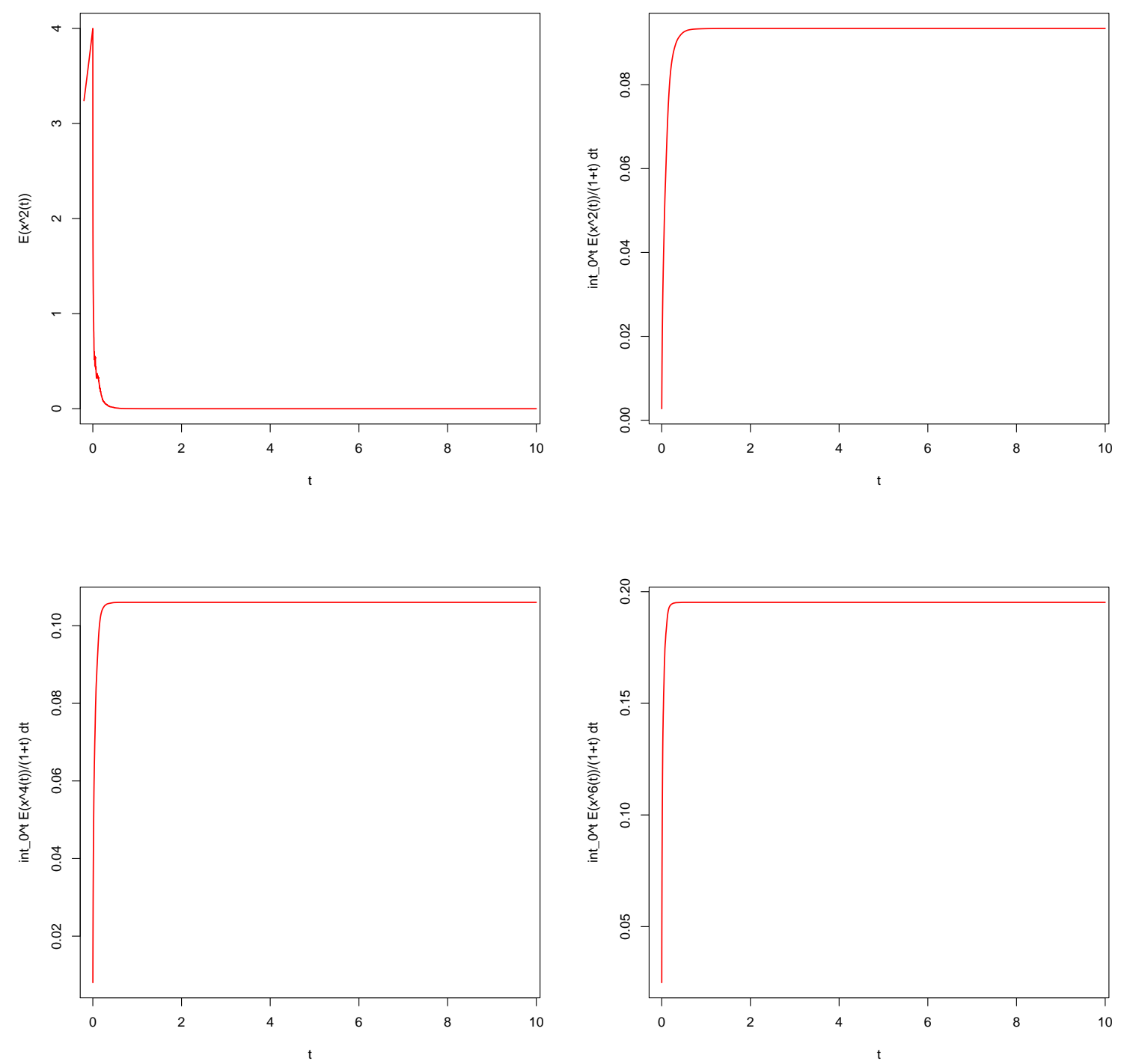

Figure 4: Simulations of $E\left(\boldsymbol{x}^{2}(t)\right), \int_{0}^{t} \frac{1}{1+t} E\left(\boldsymbol{x}^{2}(t)\right) \mathrm{d} t, \int_{0}^{t} \frac{1}{1+t} E\left(\boldsymbol{x}^{4}(t)\right) \mathrm{d} t, \int_{0}^{t} \frac{1}{1+t} E\left(\boldsymbol{x}^{6}(t)\right) \mathrm{d} t$ for the solution $\boldsymbol{x}(t)$ of system (4.1) on [0,10] and sample size 200 . 

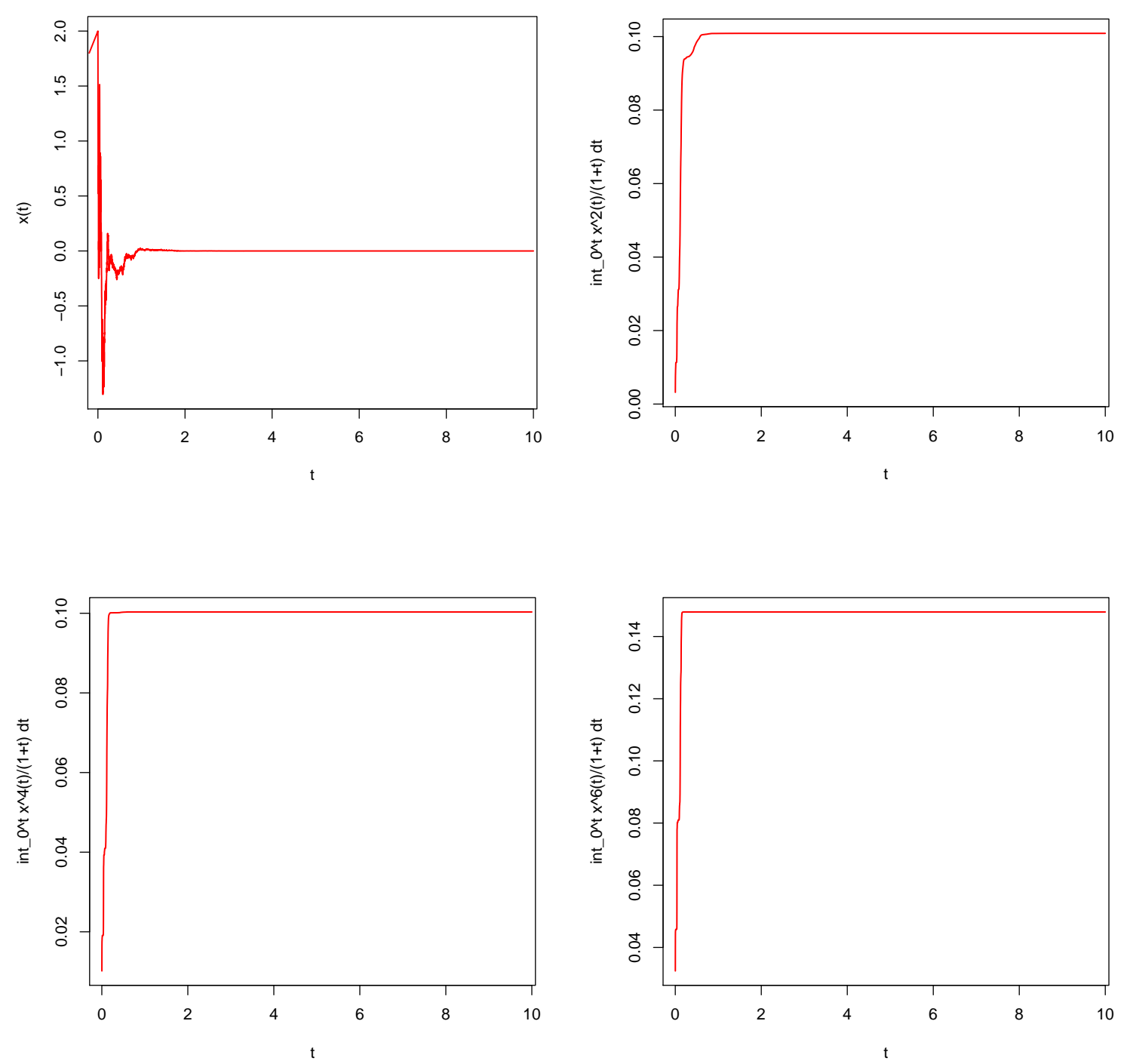

Figure 5: Simulations of the trajectories of the solution $\boldsymbol{x}(t)$ of system $(4.1), \int_{0}^{t} \frac{1}{1+t} \boldsymbol{x}^{2}(t) \mathrm{d} t$, $\int_{0}^{t} \frac{1}{1+t} \boldsymbol{x}^{4}(t) \mathrm{d} t$ and $\int_{0}^{t} \frac{1}{1+t} \boldsymbol{x}^{6}(t) \mathrm{d} t$ on $[0,10]$. 


\section{Acknowledgements}

The authors would like to thank the editor and the reviewers for their very helpful comments and suggestions. The authors would also like to thank the National Science Foundation of China (grant numbers 11571024, 31510333), the Natural Science Foundation of Hebei Province of China (A2015209229), the Leverhulme Trust (RF-2015-385), the Royal Society of London (IE131408) and the Royal Society of Edinburgh (RKES115071) for their financial support.

\section{References}

[1] G.K. Basak, A. Bisi, M.K. Ghosh, Stability of a random diffusion with linear drift, J. Math. Anal. Appl., 202: 604-622, 1996.

[2] Y. Chen, A. Xue, Improved stability criterion for uncertain stochastic delay systems with nonlinear uncertainties, Electron. Lett., 44: 458-459, 2008.

[3] L. Hu, X. Mao, Y. Shen, Stability and boundedness of nonlinear hybrid stochastic differential delay equations, Syst. Control Lett., 62: 178-187, 2013.

[4] Y. Hu, F. Wu, A class of stochastic functional differential equations with Markovian switching, J. Integral Equ. Appl., 23: 223-252, 2011.

[5] L. Huang, F. Deng, Razumikhin-type theorems on stability of stochastic retarded systems, Int. J. Syst. Sci., 40: 73-80, 2009.

[6] X. Li, X. Mao, The improved LaSalle-type theorems for stochastic differential delay equations, Stoch. Anal. Appl., 30: 568-589, 2012.

[7] R.S. Lipster, A.N. Shiryayev, Theory of Martingales, Kluwer Academic Publishers, 1989.

[8] Q. Luo, X. Mao, Y. Shen, Generalised theory on asymptotic stability and boundedness of stochastic functional differential equations, Automatica, 47: 2075-2081, 2011.

[9] X. Mao, Stochastic functional differential equations with Markovian switching, Funct. Differ. Equ., 6: 375-396, 1999.

[10] X. Mao, Robustness of stability of stochastic differential delay equations with Markovian switching, Stab. Control: Theory Appl., 3: 48-61, 2000.

[11] X. Mao, A. Matasov, A. Piunovskiy, Stochastic differential delay equations with Markovian switching, Bernoulli, 6: 73-90, 2000. 
[12] X. Mao, L. Shaikhet, Delay-dependent stability criteria for stochastic differential delay equations with Markovian switching, Stab. Control: Theory Appl., 3: 87-101, 2000.

[13] X. Mao, C. Yuan, Stochastic Differential Equations with Markovian Switching, Imperial College Press, 2006.

[14] X. Mao, Stochastic Differential Equations and Applications, 2nd ed., Horwood Publishing, Chichester, 2007.

[15] S. Peng, Y. Zhang, Some new criteria on $p$ th moment stability of stochastic functional differential equations with Markovian switching, IEEE Trans. Autom. Control, 55: 28862890, 2010.

[16] L. Shaikhet, Stability of stochastic hereditary systems with Markov switching, Theory Stoch. Process, 2: 180-184, 1996.

[17] Y. Shen, Q. Luo, X. Mao, The improved LaSalle-type theorems for stochastic functional differential equations, J. Math. Anal. Appl., 318: 134-154, 2006.

[18] M. Song, L. Hu, X. Mao, L. Zhang, Khasminskii-type theorems for stochastic functional differential equations, Discrete Contin. Dyn. Syst.-B, 18: 1697-1714, 2013

[19] S. Xu, J. Lam, X. Mao, Y. Zou, A new LMI condition for delay-dependent robust stability of stochastic time-delay systems, Asian J. Control, 7 (4): 419-423, 2005.

[20] C. Yuan, J. Lygeros, Asymptotic stability and boundedness of delay switching diffusions, IEEE Trans. Autom. Control, 51: 171-175, 2006. 